\title{
The Arf-Kervaire problem in algebraic topology: Sketch of the proof
}

\author{
Michael A. Hill, Michael J. Hopkins, and Douglas C. Ravenel
}

ABSTRACT. We provide a sketch of the proof of the non-existence of Kervaire Invariant one manifolds using equivariant homotopy theory [15]. A treatment of the statement and history of the problem can be found in "The Arf-Kervaire problem in algebraic topology: Introduction". Our goal here is to introduce the reader to the techniques used to prove the result and to familiarize them with the kinds of computations needed.

\section{Contents}

1. Introduction 2

2. Basic introduction to equivariant homotopy 3

2.1. Spheres and cells 4

2.2. Geometric fixed points and isotropy separation 5

3. The norm and the construction of $M U_{\left(C_{2} n\right)} \quad 7$

3.1. General properties of the norm 8

3.2. The spectrum $M U_{\left(C_{2} n\right)} 10$

3.3. Aside: Why not $E_{4}$ ? 11

4. Mackey functors and $R O(G)$-Graded homotopy 13

4.1. Introduction to Mackey functors 13

$\begin{array}{lll}\text { 4.2. } & R O(G) \text {-Graded homotopy groups } & 17\end{array}$

4.3. Some equivariant homotopy groups 18

5. The slice tower and the slices of $M U_{\left(C_{2} n\right)} \quad 19$

5.1. The homology of $M U_{\left(C_{2^{n}}\right)} \quad 19$

5.2. Slice filtration 21

6. The homology of a point, and the Gap theorem 26

6.1. The Bredon homology of $S^{V} \quad 27$

6.2. The Bredon homology of $S^{-V} \quad 30$

6.3. The Gap theorem 30

7. Some cohomology operations and slice differentials 32

7.1. Naming elements in Bredon homology 32

(C) 2011 International Press 
7.2. Relative cohomology operations 33

7.3. Interlude $K_{\mathbb{R}}$ and $K O \quad 36$

8. Homotopy fixed points, periodicity, and the spectrum $\Omega \quad 37$

8.1. The homotopy fixed point and periodicity theorems 38

8.2. The spectrum $\Omega$ and the detection theorem 40

References 42

\section{Introduction}

The goal of this exposition is to provide a somewhat detailed sketch of the major points of our proof of the following theorem.

TheOREM 1.1. Let $\theta_{j} \in \pi_{2^{j+1}-2} S^{0}$ be represented by a framed manifold of Kervaire invariant one. If $\theta_{j}$ is non-zero, then $j \leq 6$.

For a detailed explanation of the statement of the theorem and the history of the problem, the reader is directed to look at our historical paper [31]: "The Arf-Kervaire problem in algebraic topology: Introduction". This paper will focus on explaining the technical and equivariant aspects of the proof. In particular, we shall review some of the salient equivariant homotopy, explore the norm functor, look at particular chain complexes, and compute differentials in the slice spectral sequence. The reader is also alerted that the determination of differentials via cohomology operations $(\S 7.2)$ does not appear in the main paper; the proof here is novel. Several of the theorems (such as the Periodicity Theorem) are presented in slightly different generality as well.

A brief overview is as follows. We produce a $C_{8}$-equivariant spectrum $\tilde{\Omega}$ and prove a series of results from which the Kerviare proof follows immediately.

Detection Theorem. If $\theta_{j}$ exists, then the image in $\pi_{*} \tilde{\Omega}^{h C_{8}}$ is non-zero.

GaP TheOREM. The group $\pi_{-2} \tilde{\Omega}^{C_{8}}$ is zero.

Periodicity TheOrem. The homotopy groups of $\tilde{\Omega}^{h C_{8}}$ are 256periodic.

Homotopy Fixed Point Theorem. The spectra $\tilde{\Omega}^{C_{8}}$ and $\tilde{\Omega}^{h C_{8}}$ are weakly equivalent.

Thus we begin with an equivariant spectrum $\tilde{\Omega}$, and we produce an ordinary spectrum by taking fixed points.

Definition 1.2 . Let $\Omega$ be the $C_{8}$ fixed points of $\tilde{\Omega}$ :

$$
\Omega=\tilde{\Omega}^{C_{8}}
$$


The Homotopy Fixed Point Theorem shows that we could have equally well chosen to let $\Omega$ be the homotopy fixed points. We will sketch proofs of the Gap, Periodicity, and Homotopy Fixed Point theorems in this note. We shall not, however, prove the Detection Theorem, since this is an application of classical techniques of a somewhat different flavor than the rest. This has a slight expository difficulty related to which classes one must invert to produce our spectrum $\tilde{\Omega}$. We focus here on the close connection with the Lubin-Tate spectrum $E_{4}$, but even still, the flavor of $\S 8.2$ is different. The interested reader is referred to the main paper for a proof of the Detection Theorem, the specifics of the inverted classes, and a more detailed treatment of the theorems sketched herein $[\mathbf{1 5}]$.

\section{Basic introduction to equivariant homotopy}

Though we will never make it explicit, we shall assume we are working in a rigid point-set model category whose homotopy theory is the usual homotopy theory of equivariant spectra $[\mathbf{2 0}, \mathbf{2 2}, \mathbf{2 3}]$. In particular, we shall suppress any assumptions about fibrancy or cofibrancy of spectra in various constructions, and we shall focus on the most important aspects of the theory. The reader is suggested to consult the survey articles referenced here for more details about the general theory $[\mathbf{5 , 2 4}]$.

There are many different (and inequivalent) notions of $G$-spectra. For our purposes, we consider "honest" $G$-spectra. These are $G$-spectra indexed by finite subspaces of an infinite dimensional inner product space that contains every irreducible representation of $G$ infinitely often. The model categorical notions will not be explicitly spelled out, and instead we will focus on the computational aspects.

We begin with some standard notation. We will let $\mathcal{S}_{G}$ denote any of the nice categories of equivariant spectra. If $X$ is a $G$-spectrum, then we shall also denote by $X$ the image of the restriction functor to any subgroup $H$ of $G$. The category of $G$-spectra is tensored and cotensored over $G$-spaces, and the function spectra objects are again $G$-spectra.

If $X$ and $Y$ are $G$-spectra, then we shall let $[X, Y]$ denote the homotopy classes of $G$-equivariant maps from $X$ to $Y$. This is the same as $\pi_{0}$ of the $G$-fixed points of $F(X, Y)$. If we there is potential for confusion, we will adorn $[X, Y]$ with a subscript $G$ to make clear the group. For a general $G$-space or spectrum $X$, we will let $X^{G}$ denote the fixed point object.

We now recall that the forgetful functor $\mathcal{S}_{G} \rightarrow \mathcal{S}_{H}$ has a left and a right adjoint. The left adjoint, normally written $G_{+} \wedge_{H}-$ is the "induction" functor, and we have a natural isomorphism

$$
G_{+} \wedge_{H} X \cong G / H_{+} \wedge X
$$

whenever $X$ is a $G$-spectrum. The right adjoint, written $F_{H}(G,-)$ is the "coinduction" functor. One of the most delightful aspects of the theory is that for $G$ a finite group, these are actually the same. 
THEOREM 2.1 (Wirthmüller [30]). We have a natural equivalence

$$
G_{+} \wedge_{H} X \cong F_{H}(G, X) .
$$

We should think of this as the equivariant analogue of the statement that in spectra, finite coproducts $\left(G_{+} \wedge_{H} X\right)$ are the same as finite products $\left(F_{H}(G, X)\right)$. For this reason, coinduction will not be talked about again.

This Wirthmüller isomorphism gives rise to natural isomorphisms

$$
\left[G_{+} \wedge_{H} X, Y\right]_{G} \cong[X, Y]_{H} \cong\left[X, F_{H}(G, Y)\right]_{G} \cong\left[X, G_{+} \wedge_{H} Y\right]_{G} .
$$

2.1. Spheres and cells. Given an orthogonal representation of $V$, we can form two spheres: $S(V)$, the unit sphere in $V$, and $S^{V}$, the one point compactification of $V$. These are related by a cofiber sequence in spaces

$$
S(V)_{+} \rightarrow S^{0} \rightarrow S^{V},
$$

making $S^{V}$ the unreduced suspension of $S(V)$. We remark that for every representation $V, S^{V}$ has at least 2 fixed points: the origin and the point at infinity. The inclusion of these points will play a very important role in our proof.

For now, we fix some notation for the representations which will appear.

Definition 2.2. Let $\sigma$ denote the 1-dimensional sign representation of $C_{2^{n}}$.

Let $\lambda: C_{p^{n}} \rightarrow S^{1}$ denote the inclusion of the $p^{n}$ th roots of unity.

Let $\lambda(k)$ denote the composite of $\lambda$ with the $k^{\text {th }}$ power map on $S^{1}$.

Let $\rho_{2^{n}}$ denote the regular representation of $C_{2^{n}}$, and let $\bar{\rho}_{2^{n}}$ denote the quotient by the trivial representation.

If we work localized at $p$, then $S^{\lambda(k)}$ is indistinguishable from $S^{\lambda\left(k^{\prime}\right)}$ whenever the $p$-adic valuation of $k$ and $k^{\prime}$ agree. For this reason, we will restrict attention to the collection $\lambda\left(p^{r}\right)$. Since there is a $\sigma$ for every cyclic 2 -group, when we need to distinguish the sign representation for $C_{2^{k}}$, we will denote it by $\sigma_{k}$.

Although this gives us two very good notions of spheres, our notion of cells will be somewhat more elementary: we induce up from the standard notions of cells. Our $n$-cells are those $G$-spectra of the form $G_{+} \wedge_{H} D^{n}$, where $D^{n}$ is given the trivial $H$-action. Since $D^{n}$ with the trivial $H$-action is visibly the restriction of $D^{n}$ with the trivial $G$-action, we consider only cells of the form

$$
G / H_{+} \wedge D^{n}
$$

The boundary is of course $G / H_{+} \wedge S^{n-1}$, and we build our notion of CWcomplex with these as our cells. Additionally, understanding how to attach to $X$ cells of the form $G / H_{+} \wedge D^{n}$ amounts to understanding

$$
\left[G / H_{+} \wedge S^{n-1}, X\right]_{G} \cong\left[S^{n-1}, X\right]_{H} \cong \pi_{n-1}\left(X^{H}\right) .
$$

These kinds of homotopy groups form a rich structure and will be spelled out in more detail in $\S 4$. 
2.2. Geometric fixed points and isotropy separation. Perhaps the most difficult and in many ways counter-intuitive aspect of equivariant homotopy theory is the notion of "fixed points". In general, the fixed point functor from $G$-Spectra to ordinary spectral is very badly behaved. It is not symmetric monoidal, and it doesn't commute with infinite suspension. Even if the most elementary cases, understanding the fixed points is a non-trivial undertaking. For example, the fixed points for the sphere with a trivial $G$-action are shockingly complicated $[\mathbf{8}, \mathbf{7}]$.

TheOREM 2.3 (tom Dieck). As rings, $\pi_{0}\left(S^{0}{ }^{G}\right)$ is the Burnside ring of finite $G$-sets $A(G)$.

Already this is much more complicated that what we would expect the fixed points to be, namely $S^{0}$. This is also reflected in a splitting of the fixed points into a wedge of more complicated spectra.

Equivariant homotopy theory has machinery to correct this: geometric fixed points.

THEOREM 2.4. There is a lax symmetric monoidal functor

$$
\Phi^{G}: \mathcal{S}_{G} \rightarrow \mathcal{S}
$$

such that

$$
\Phi^{G}\left(\Sigma^{\infty} X_{+}\right)=\Sigma^{\infty} X_{+}^{G} .
$$

In other words, the geometric fixed point functor behaves exactly the way our intuition for fixed points suggests.

Corollary 2.5. As rings, $\pi_{0}\left(\Phi^{G}\left(S^{0}\right)\right)=\mathbb{Z}$, and for a general virtual representation $V, \Phi^{G}\left(S^{V}\right)=S^{V^{G}}$.

The definition of $\Phi^{G}$ will actually prove surprisingly useful. We begin with the notion of a classifying space and universal space for a family of subgroups of a group. Let $\mathcal{F}$ denote a family of subgroups of $G$ such that if $H \in \mathcal{F}$, then all subgroups and conjugates of $H$ are also in $\mathcal{F}$.

Definition 2.6. A universal space associated to $\mathcal{F}$ is a $G$-space $E \mathcal{F}$ such that

$$
(E \mathcal{F})^{H} \simeq \begin{cases}* & H \in \mathcal{F} \\ \emptyset & H \notin \mathcal{F} .\end{cases}
$$

Thus the restriction of $E \mathcal{F}$ to any subgroup $H \in \mathcal{F}$ is $H$-equivariantly contractible. That such a space exists for a general group follows by mirroring Milnor's join construction of $E G$. For cyclic $p$-groups, we can find a distinguished representative $E \mathcal{F}$ for any family $\mathcal{F}$.

Proposition 2.7. Let $\mathcal{F}_{m}$ be the collection of proper subgroups of $C_{p^{m}} \subset$ $C_{p^{n}}$, and let $E \mathcal{F}_{m}$ denote the usual universal space. Then

$$
E \mathcal{F}_{m} \simeq \lim _{\rightarrow} S\left(k \lambda\left(p^{m-1}\right)\right),
$$

the "unit sphere" in the infinite direct sum of copies of $\lambda\left(p^{m-1}\right)$. 
PROOF. If we restrict $\lambda\left(p^{m-1}\right)$ to any proper subgroup of $C_{p^{m}}$, then we get the trivial representation. Similarly, the only fixed points for the $C_{p^{m}}$ action is the origin. We therefore conclude that

$$
S\left(k \lambda\left(p^{m-1}\right)\right)^{C_{p^{\ell}}}= \begin{cases}S^{2 k-1} & \ell<m \\ \emptyset & \ell \geq m .\end{cases}
$$

The maps in the colimit are the standard inclusions $S^{2 k-1} \rightarrow S^{2 k+1}$, and so the colimit is contractible.

Having built $E \mathcal{F}$, we can now build a much more important space: $\tilde{E} \mathcal{F}$. This is by definition the cofiber of the canonical map $E \mathcal{F}_{+} \rightarrow S^{0}$, in spaces. As a consequence of the previous proposition, we can also identify a representative of the homotopy type $\tilde{E} \mathcal{F}_{m}$ for cyclic $p$-groups.

Proposition 2.8. A model for $\tilde{E} \mathcal{F}_{m}$ is given by

$$
\lim _{\rightarrow} S^{k \lambda\left(p^{m-1}\right)}
$$

REMARK 2.9. In both of these propositions, the actual representations used don't matter. What mattered was that the fixed points of the proper subgroups become increasingly highly connected (which means we had an increasing number of copies of $\lambda\left(p^{\ell}\right)$ where $\left.\ell \geq m-1\right)$ and that there were no fixed points for $C_{p^{m}}$ (which means that we have no copies of $\lambda\left(p^{\ell}\right)$ for $\ell \geq m)$. We could, however, have added arbitrarily many copies of $\lambda\left(p^{k}\right)$ for $k<m$ and still produced the same homotopy type.

Though we will use several variants of $\tilde{E} \mathcal{F}$, by far the most important is when $\mathcal{F}$ is the family of all proper subgroups of $G$. In this case, $E \mathcal{F}_{+} \rightarrow S^{0}$ is an equivalence when restricted to any proper subgroup, and $S^{0} \rightarrow \tilde{E} \mathcal{F}$ is an equivalence on $G$-fixed points.

Definition 2.10. Let $\mathcal{F}$ denote the family of proper subgroups of $G$. If $X$ is a $G$-spectrum, then smashing $X$ with the cofiber sequence defining $\tilde{E} \mathcal{F}$ yields the isotropy separation sequence:

$$
E \mathcal{F}_{+} \wedge X \rightarrow X \rightarrow \tilde{E} \mathcal{F} \wedge X
$$

The geometric fixed points of $X$ are the $G$-fixed points of $\tilde{E} \mathcal{F} \wedge X$ :

$$
\Phi^{G}(X)=(\tilde{E} \mathcal{F} \wedge X)^{G} .
$$

Both parts of this definition will be vitally useful. The isotropy separation sequence does exactly as the name implies: it separates $X$ into parts that are built from cells of the form $G / G_{+} \wedge D^{n}$ (the $\tilde{E} \mathcal{F} \wedge X$ part) and those that are built from induced cells (the $E \mathcal{F}_{+} \wedge X$ part). Thus when we compute with this, the left most term is often handled by induction on the 
order of $G$ (since all of these cells are induced). We can make this more precise. By the defining cofiber sequence for $\tilde{E} \mathcal{F}$, any map

$$
G / H_{+} \wedge Y \rightarrow \tilde{E} \mathcal{F} \wedge X
$$

is automatically null-homotopic (the target is contractible when restricted to $H)$.

Additionally, the map

$$
E \mathcal{F}_{+} \wedge X \rightarrow X
$$

is a kind of generalized restriction, while $X \rightarrow \tilde{E} \mathcal{F} \wedge X$ is a localization. We can use this intuition to provide some heuristic justification for tom Diek's theorem. If we apply fixed points and take $\pi_{*}$ then we have a long exact sequence (where we use $\Phi^{G}\left(S^{0}\right)=S^{0}$ ):

$$
\cdots \rightarrow \pi_{1}\left(S^{0}\right) \rightarrow \pi_{0}\left(\left(\Sigma^{\infty} E \mathcal{F}_{+}\right)^{G}\right) \rightarrow \pi_{0}\left(S^{0}{ }^{G}\right) \rightarrow \pi_{0}\left(S^{0}\right) \rightarrow 0 .
$$

If we can understand the homotopy of $\left(\Sigma^{\infty} E \mathcal{F}_{+}\right)^{G}$, then we can compute $\pi_{0}\left(S^{0}\right)$. However, since we are working stably, we have stable transfer maps in $\pi_{0}$ arising from the obvious map

$$
G / H_{+} \wedge X \rightarrow X
$$

In this context, this gives us a map

$$
\pi_{0}\left(S^{0^{H}}\right)=\pi_{0}\left(G / H_{+} \wedge \Sigma^{\infty} E \mathcal{F}_{+}\right) \rightarrow \pi_{0}\left(\left(\Sigma^{\infty} E \mathcal{F}_{+}\right)^{G}\right)
$$

Induction on $G$ then gives the result. We remark that though it is not shown here, all of the groups in question (with the exception of $\pi_{1} S^{0}$ ) are actually finite rank free abelian groups. This can also essentially be shown by downward induction on the order of the group.

\section{The norm and the construction of $M U_{\left(C_{2} n\right)}$}

One of the most useful constructions in our main paper is that of the norm. This is a multiplicative induction that takes $\mathcal{S}_{H}$ to $\mathcal{S}_{G}$ whenever $H \subset G$, and it generalizes the Evens' transfer in group cohomology [11]. In the homotopical context, it was studied by Greenlees-May [14]. This section serves as a very general overview, giving intuition and some algebraic constructions that will help the reader understand the basic properties needed for the proof. In the main paper, a detailed construction with the proofs of the necessary equivariant properties is provided. 
3.1. General properties of the norm. The basic idea of the norm is simple: induction in a category is essentially the coproduct of an object indexed by cosets. For representations, this is simply saying that we take the direct sum of $|G / H|$-copies of the representation and permute them in the obvious way, and for spectra, we instead use the wedge. In both of these examples, the underlying object is just the iterated coproduct, and the equivariance can be read out of the permutation action on $G / H$ and the $H$-action on the underlying object.

Commutative algebras and $E_{\infty}$-ring spectra have a different coproduct: the tensor or smash product. In these cases, again have a kind of induction, the norm. The two constructions are quite similar. We will sketch the one for commutative rings, and then state the salient properties of the one for spectra.

Proposition 3.1. There is a multiplicative functor $N_{H}^{G}: \mathcal{A l} g_{H} \rightarrow \mathcal{A l} g_{G}$ which is left adjoint to the forgetful functor $i_{H}$. The composite $i_{H} \circ N_{H}^{G}$ is the $|G / H|$-fold tensor power functor when $H$ is a normal subgroup.

In fact, the construction is obvious. The underlying algebra for $N_{H}^{G}(A)$ is

$$
\bigotimes_{G / H} A
$$

To describe the equivariance, we chose a set of coset representative $g_{i}$ (and we chose one of them to be the identity element). For any $g \in G$ and for all $i, g g_{i}=g_{j} h$ for some $j$ and $h$. This allows us to specify the action: $g$ acts on the factor indexed by $g_{i}$ by sending it to the factor indexed by $g_{j}$ and acting by $h$.

We can best understand the norm on the free commutative algebra on an $H$-set $X$, namely the polynomial algebra on $X, \mathbb{Z}[X]$.

Proposition 3.2. If $X$ is an $H$-set, then

$$
N_{H}^{G}(\mathbb{Z}[X])=\mathbb{Z}\left[G \times_{H} X\right] .
$$

This shows immediately the desired adjunctions. It also shows the failure of the restriction to simply be the tensor product of the underlying algebras. If $H$ is not normal, then the restricting of $G \times_{H} X$ to $H$ is not necessarily the disjoint union of copies of $X$.

It should be noted now that the norm functor actually lives as one on $H$-modules, not just $H$-algebras. On the $H$-algebras, it has a particularly nice description as a left adjoint. On the others, it's just the tensor power with the permutation $G$-action.

All of these statements are also true for commutative ring objects in $H$-spectra, namely the $E_{\infty}$-ring spectra. Let $\operatorname{Comm}_{G}$ denote the category of $G$-equivariant $E_{\infty}$ ring spectra. 
Proposition 3.3. The forgetful functor $i_{H}:$ Comm $_{G} \rightarrow$ Comm $_{H}$ has a left adjoint $N_{H}^{G}$, the underlying $H$-spectrum for which is the $|G / H|$-fold smash power when $H$ is normal.

The composite of the norm with the restriction as an endofunctor of Comm $_{G}$ is also understandable:

$$
N_{H}^{G}(X)=G / H_{+} \otimes X,
$$

where we have used that $E_{\infty} G$-spectra are tensored over pointed $G$-sets.

Just as for commutative rings, there is a prolongation of this functor to all $H$-spectra. It will be essential to our proof of the Periodicity Theorem to have some computational results about it. These all rely on the following immediate fact.

Proposition 3.4. If $W$ is a virtual representation of $H$, then $N_{H}^{G} S^{W}=$ $S^{\operatorname{Ind} d_{H}^{G} W}$.

This has the following very useful consequence. If $f: S^{W} \rightarrow X$ is an $H$-equivariant map, then we have a $G$-equivariant map

$$
N_{H}^{G}(f): S^{I n d_{H}^{G} W} \rightarrow N_{H}^{G}(X) .
$$

In particular, if $X$ is the restriction of a $G$-equivariant $E_{\infty}$-ring spectrum, then we can compose with the counit of the adjunction to get a new $G$-equivariant map

$$
N_{H}^{G}(f): S^{I n d_{H}^{G} W} \rightarrow X .
$$

This gives us an additional, multiplicative way to promote $H$-equivariant homotopy classes to $G$-equivariant ones. Similarly, it is often quite simple to check that these are non-zero by restriction. If $H$ is normal in $G$, then the restriction of $N_{H}^{G}(f)$ is essentially the $|G / H|$-fold power of $f$.

One of the most important properties of the norm is that it is wellbehaved with respect to geometric fixed points. Since $S^{0}=N_{e}^{G} S^{0}$, we expect terrible behavior for actual fixed points, and much of our work is spent boot-strapping up from the geometric fixed points.

THEOREM 3.5. If $X$ is an $H$-equivariant spectrum, then

$$
\Phi^{G}\left(N_{H}^{G}(X)\right) \simeq \Phi^{H}(X) .
$$

A very coarse sketch of the argument is as follows. We know from the earlier propositions that if $X=S^{V}$ and if $V_{0}=V^{H}$, then

$$
\Phi^{G} N_{H}^{G}\left(S^{V}\right) \simeq \Phi^{G} S^{I n d_{H}^{G} V} \simeq S^{V_{0}} \simeq \Phi^{H} S^{V} .
$$

Now if we build $X$ out of cells, then we have a diagram for building $N_{H}^{G}(X)$ out of cells. What is essential here is that the group $G$ is acting on the diagram. The only non-induced cells in the diagram are those of the form $N_{H}^{G}(e)$, where $e$ is a cell of $X$. Since geometric fixed points destroys induced cells, we are reduced to the case of spheres. 
3.2. The spectrum $M U_{\left(C_{2}\right)}$. A central role in our proof is played by the honest $C_{2}$-equivariant spectrum of Real bordism $M U_{\mathbb{R}}[\mathbf{2}, \mathbf{1 9}]$. This is the bordism spectrum of $C_{2}$-equivariant manifolds together with a Real structure on their stable normal bundle. This is a $C_{2}$-equivariant vector bundle where the fibers are complex vector spaces and where on fibers, the $C_{2}$-action is conjugate linear. With this in mind, the underling spectrum is just $M U$ together with its complex conjugation action. If we take geometric fixed points (which corresponds heuristically to passage to fixed points for the Real manifolds), then we recover the unoriented bordism spectrum $M O$. These facts are central to our understanding of the spectrum.

Numerous authors have studied $M U_{R}$, computing its homotopy groups, showing that its fixed and homotopy fixed points agree, and determining its slices $[\mathbf{1}, \mathbf{1 7}, \mathbf{1 8}]$. Additionally, we know that this is a $C_{2}$-equivariant $E_{\infty}$-ring spectrum, so the norm will let us build spectra for any finite group $G$ containing $C_{2}$ that have similar universal properties.

Definition 3.6. Let $M U_{(G)}$ denote $N_{C_{2}}^{G} M U_{\mathbb{R}}$.

By Theorem 3.5, we know also that

$$
\Phi^{G} M U_{(G)}=M O .
$$

The spectrum $M U_{\left(C_{2^{n}}\right)}$ is also an equivariant Thom spectrum. It carries geometric information of manifolds together with " $2^{n-1}$ st roots of a Real structure". We will not exploit this geometry here, though a better understanding would give another proof of our Reduction theorem.

Just as in the ordinary case, $M U_{\mathbb{R}}$ carries orientations. Classically these are maps from $M U$, and in the $C_{2}$-equivariant case, these are maps from $M U_{\mathbb{R}}$. Many familiar spectra with natural $C_{2}$-actions have equivariant refinements, and their underlying complex orientation also refines to a Real one. In particular, $K$-theory becomes Atiyah's Real $K$-theory $K_{\mathbb{R}}$, and the standard orientation of $K$-theory becomes a real orientation of $K_{\mathbb{R}}$ [3]. Generalizing this, ongoing work of Avarett essentially shows that the push-forwards of the Lubin-Tate spectra $E_{n}$ (endowed with their HopkinsMiller $C_{2}$-action [26]) admit Real orientations [4]. This generalizes in the following way.

Proposition 3.7. If $E$ is a $G$-equivariant $E_{\infty}$ ring spectrum for $G$ a finite group containing $C_{2}$, and if the restriction of $E$ to $C_{2}$ admits a Real orientation

$$
u: M U_{(G)} \rightarrow E
$$

then there is a canonical equivariant $E_{\infty}$ map

$$
N(u): M U_{(G)} \rightarrow E .
$$

Proof. This follows immediately from the adjunction

$$
\operatorname{Map}_{\operatorname{Comm}_{G}}\left(M U_{(G)}, E\right)=\operatorname{Map}_{\operatorname{Comm}_{C_{2}}}\left(M U_{\mathbb{R}}, E\right) .
$$

Our desired map is the image of the orientation. 
Thus $E_{2^{n-1}}$ admits a canonical map from $M U_{\left(C_{2^{n}}\right)}$ that lifts the Real orientation.

3.3. Aside: Why not $E_{4}$ ? We pause here to explain part of the proof, namely why we do not use $E_{4}$. In fact, the Detection Theorem shows that the $C_{8}$-homotopy fixed points of $E_{4}$ is sufficient to detect the Kervaire classes. We know from the Nilpotence Theorem that there is a horizontal vanishing line in the homotopy fixed points spectral sequence $[\mathbf{6}]$, and that tells us immediately that the homotopy groups are periodic with period some power of 2 . We can approach this by running the spectral sequence and seeing what classes on the zero line survive or by determining what sorts of bundles are orientable for $E_{4}^{h C_{8}}$ (which tells us immediately which classes must be permanent cycles).

However, even in the toy case of $C_{4}$ acting on $E_{2}$, it is very difficult to determine the differentials and extensions in the homotopy fixed point spectral sequence. Figure 1 shows the $E_{5}$ term of the spectral sequence (with an infinite number of copies of $\pi_{*} b o$ suppressed).

The spectral sequence terminates at $E_{14}$, and at that point, we can see that it is 32 -periodic. What is not obvious from the $E_{2}$-term is that $\pi_{-2} E_{2}^{h C_{4}}=0$. Determining the differentials on the class denoted by a star is one of the trickier parts of this computation. In fact, understanding the

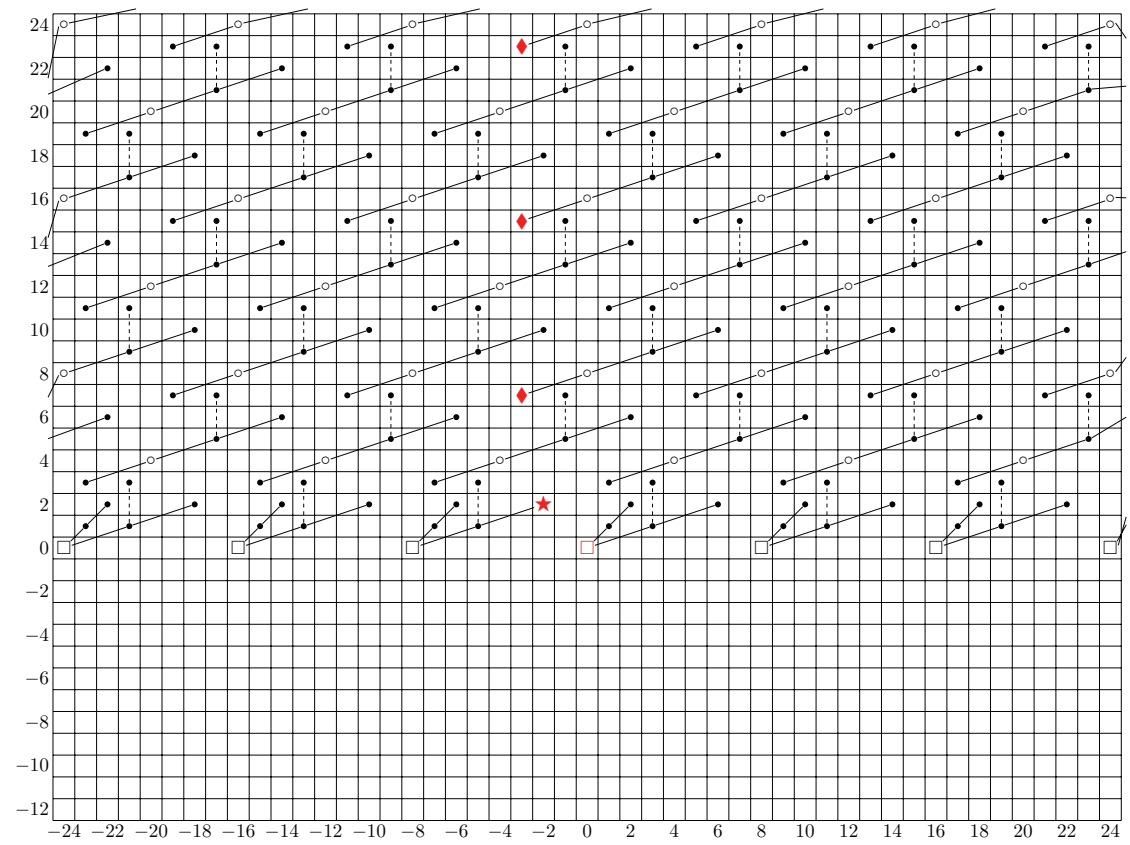

Figure 1. The $E_{5}$ term of the Homotopy Fixed Point Spectral Sequence for $E_{2}^{h C_{4}}$ 
analogue of the Kervaire differential required knowledge of some multiplicative relations in $\pi_{28} S^{0}$ at 2 .

The $C_{8}$ case was visibly harder. While the $C_{4}$ case could be nicely presented at $E_{5}$ (in that each symbol represents either $\mathbb{Z}$ or a finite group), after much analysis with the slice spectral sequence, we believe that the $C_{8}$ case will not have a nice picture until $E_{16}$. Additionally, we know there will be a great many differentials before then, since there are fairly complicated patterns of differentials for $C_{2}$ and $C_{4}$ acting on $E_{4}$. It became clear early on that running the homotopy fixed point spectral sequence for $E_{4}^{C_{8}}$ would suffer from two major drawbacks:

(1) It would be very difficult to determine and to justify all differentials and extensions in the spectral sequence, and

(2) No one would ever believe it.

This led us to consider honest fixed points, rather than homotopy ones. While this might appear to be a Faustian bargain, trading rigidity for uncomputability, we had a major break-through with the creation of the slice spectral sequence. This provides a filtration of equivariant homotopy theory for a finite group $G$, and while the full form will not appear in this paper, this is the filtration of $M U_{\left(C_{2^{n}}\right)}$ that makes computations doable. We should think of this as a much more tractible Atiyah-Hirzebruch spectral sequence. This filtration has a couple of antecedents: Dugger built the $C_{2}$-equivariant form and showed that $K_{\mathbb{R}}$ has the expected filtrations quotients and Hopkins-Morel, Voevodsky built an analogous filtration in the motivic context $[\mathbf{1 6}, \mathbf{2 7}, \mathbf{2 8}, \mathbf{2 9}]$.

The slice spectral sequence computes the honest fixed points of an equivariant spectrum (in fact, it does much more: since it is a purely equivariant filtration, it computes the equivariant homotopy groups as a Mackey func-

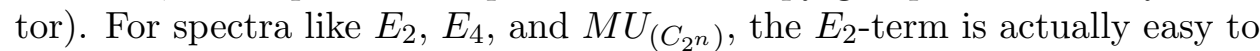
compute. In Figure 2, we show the $E_{5}$-page of the slice spectral sequence for $C_{4}$ acting on $E_{2}$.

Comparing with Figure 1 shows some obvious advantages. It is immediate that $\pi_{-2}$ of the fixed points is zero, since it is zero on $E_{5}$. In fact, it is zero on $E_{2}$, and the Gap Theorem below shows that it is zero for a large family of equivariant spectra. The rigidity of equivariant homotopy theory helps us produce a large collection of permanent cycles in the fixed points, and these give us our desired periodicity.

The obvious advantage of the slice spectral sequence necessitates proving that the slices of $E_{4}$ have the required form. This in turn required first understanding the slices of $M U_{\left(C_{2}\right)}$ and for various localizations thereof. It became clear that a judicious choice of localization of $M U_{\left(C_{2^{n}}\right)}$ would sit as an intermediary between $M U_{\left(C_{2^{n}}\right)}$ and $E_{4}$, being sufficiently strong to detect the Kervaire classes but also simple to work with. The reader is suggested to keep the spectrum $E_{4}$ with its natural $C_{8}$-action in mind, especially in $\S 8$, where the intuition provided by $E_{4}$ is exactly correct. 


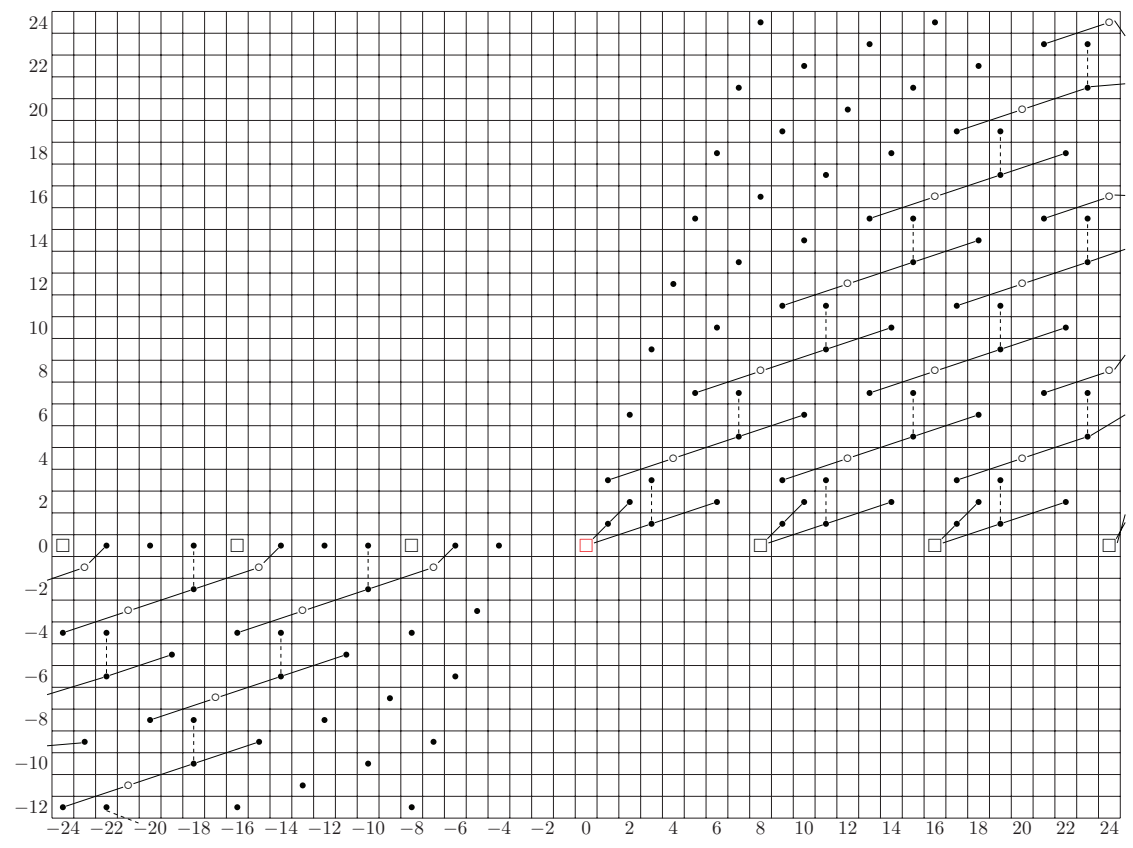

Figure 2 . The slice $E_{5}$-term for $C_{4}$ acting on $E_{2}$

\section{Mackey functors and $R O(G)$-Graded homotopy}

4.1. Introduction to Mackey functors. One of the biggest hurdles facing homotopy theorists interested in equivariant algebraic topology is the more complicated notion of homotopy groups. Classically, the homotopy groups of a spectrum are simply abelian groups. In the equivariant context, the homotopy groups are objects in a more complicated abelian category, the category of Mackey functors [9]. The complexity, and the definition of Mackey functors, arises from the larger collection of "points" in the equivariant context. In addition to $*=G / G$, we have any coset space $G / H$. These $G$-sets are linked by a large collection of stable $G$-maps, and these provide a collection of maps between the homotopy groups.

Definition 4.1. Let $\mathcal{O}_{G}$ denote the category of finite $G$-sets.

Definition 4.2. A Mackey functor $\underline{M}$ is a pair of functors $M^{*}: \mathcal{O}_{G}^{o p} \rightarrow$ $\mathcal{A} b$ and $M_{*}: \mathcal{O}_{G} \rightarrow \mathcal{A} b$ which send disjoint unions to direct sums, such that

$$
M^{*}(X)=M_{*}(X)=: \underline{M}(X)
$$

for all objects $X$, and such that if

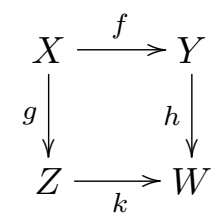


is a pull-back square in $G$-sets, then

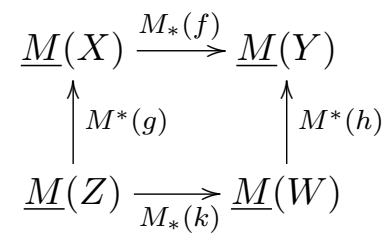

is commutative.

We will denote $M^{*}(f)$ by $f^{*}$ and $M_{*}(f)$ by $f_{*}$, and we will call $f^{*}$ a restriction and $f_{*}$ a transfer. If $f: G / H \rightarrow G / G$ is the obvious map, then following this we will often write $\operatorname{Tr}_{H}^{G}$ for $f_{*}$ and $\operatorname{Re} s_{G}^{H}$ for $f^{*}$. This terminology will be made somewhat more clear by connecting these to standard constructions in homotopy theory.

Definition 4.3. If $X$ is a $G$-spectrum, then the $k^{\text {th }}$ homotopy group of $G$ is the Mackey functor

$$
\underline{\pi_{k}(X)}(B)=\left[B_{+} \wedge S^{k}, X\right]_{G}
$$

When $B=G / H$, we will also let

$$
\pi_{k}^{H}(X)=\underline{\pi_{k}(X)}(B) .
$$

This description makes the restriction maps immediate: they arise by precomposition. For the transfer maps, we can work one of two ways.

(1) In the equivariant stable homotopy category, associated to a map $\Sigma^{\infty} B_{+} \rightarrow \Sigma^{\infty} C_{+}$there is a transfer map $\Sigma^{\infty} C_{+} \rightarrow \Sigma^{\infty} B_{+}$. Precomposing with these transfer maps gives the desired transfer maps.

(2) Using the isomorphisms

$$
\left[G / H_{+} \wedge X, Y\right]_{G} \cong[X, Y]_{H} \cong\left[X, G / H_{+} \wedge Y\right]_{G},
$$

we can conclude that there is a natural isomorphism

$$
\left[B_{+} \wedge X, Y\right] \cong\left[X, B_{+} \wedge Y\right]
$$

for all $G$-sets $B$ and for all $X$ and $Y$. We can therefore consider the compositions in the target, rather than in the source, to get our transfer maps.

Since the object function is assumed to be additive, it suffices to understand what happens on the orbits $G / H$ :

$$
\pi_{k}^{H}(X)=\left[G / H_{+} \wedge S^{k}, X\right]_{G}=\left[G_{+} \wedge_{H} S^{k}, X\right]_{G}=\left[S^{k}, X\right]_{H}=\pi_{k}\left(X^{H}\right) .
$$

Thus the value of the homotopy group Mackey functor on $G / H_{+}$is exactly $\pi_{k}$ of the $H$-fixed points of $X$. Moreover, if $H \subset K \subset G$, then we have a $G$-map $G / H \rightarrow G / K$ which gives us a map in the other way on the Mackey functor homotopy groups. This coincides with the restriction 
map $\pi_{k}\left(X^{K}\right) \rightarrow \pi_{k}\left(X^{H}\right)$ induced by inclusion of fixed points. Similarly, the transfer map is essentially "summing over the cosets".

Since they are a diagram category of abelian groups, Mackey functors form an abelian category. This abelian category is closely tied to the equivariant stable homotopy category.

Proposition 4.4. Let $\mathcal{S}_{G, 0}$ denote the full subcategory of $\mathcal{S}_{G}$ consisting of those objects $X$ such that $\pi_{i}(X)=0$ for $i \neq 0$. Then $\pi_{0}$ induces an equivalence of categories between the homotopy category of $\overline{\mathcal{S}_{G, 0}}$ and the category of Mackey functors.

In particular, for any Mackey functor $\underline{M}$, we have an associated Eilenberg-Mac Lane spectrum $H \underline{M}$ which satisfies

$$
\underline{\pi_{k}(H \underline{M})}= \begin{cases}\underline{M} & k=0, \\ 0 & \text { otherwise. }\end{cases}
$$

We will be most concerned with $H \underline{\mathbb{Z}}$.

Just as with abelian groups, we can define "bilinear" pairings of two Mackey functors $\underline{M}$ and $\underline{N}$ into a third, $\underline{P}$. This is a natural transformation $M^{*} \otimes N^{*} \rightarrow P^{*}:$

$$
\langle-,-\rangle_{X}: \underline{M}(X) \otimes \underline{N}(X) \rightarrow \underline{P}(X)
$$

together with the condition that for any $f: X \rightarrow Y$, the following diagram commutes

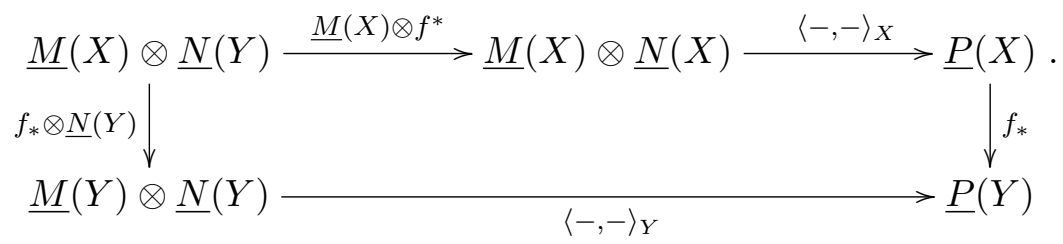

Put more sucinctly:

$$
\langle\operatorname{Tr}(x), y\rangle_{Y}=\operatorname{Tr}\left(\langle x, \operatorname{Res}(y)\rangle_{X}\right) .
$$

A Green functor is a Mackey functor $\underline{M}$ together with a bilinear pairing $\underline{M} \otimes \underline{M} \rightarrow \underline{M}$. Since this definition is opaque, we will unpack it slightly. A Green functor $M$ is one for which $\underline{M}(X)$ is a ring for all $X$ and for which all restriction maps $f^{*}$ are ring homomorphisms. This means that for any $f: X \rightarrow Y, \underline{M}(X)$ is a $\underline{M}(Y)$-module via $f^{*}$, and the condition for the pairing can be restated as follows: $f_{*}$ is a map of $\underline{M}(Y)$-modules. It is then immediate how one defines modules over a Green functor.

The most important Green functor is the Burnside ring $\underline{A}$. This associates to a finite $G$-set $X$ the Grothendieck group of finite $G$-sets over $A$, and restriction and transfer are just pull-back and composition. The pullback of two $G$-sets over $X$ gives a product on this set, making $\underline{A}(X)$ into a ring for all $X$. It is then trivial to check that transfers are module maps over 
the restrictions. It is immediate that $\underline{A}(G / G)=A(G)$, the Burnside ring of finite $G$-sets. If $H$ is a subgroup of $G$, then the map

$$
(f: X \rightarrow G / H) \mapsto\left(f^{-1}(e H) \rightarrow e H\right)
$$

identifies $\underline{A}(G / H)$ with $\underline{A}(H)$. In particular, $\underline{A}(G /\{e\})=\mathbb{Z}$.

The Burnside Mackey functor plays the role for Mackey functors that $\mathbb{Z}$ plays for abelian groups. In particular, every Mackey functor is a module over the Burnside ring Mackey functor. The module structure is actually very easy to spell out: transfers are module maps. If we look at a particular orbit $G / H$, then this is the transfer, in the Burnside ring, of the element $1 \in A(H)$. By the defining property of a pairing of Mackey functors, we then know how it acts on an element $x \in \underline{M}(G / G)$ :

$$
[G / H] \cdot x=\operatorname{Tr}_{H}^{G} \operatorname{Res}_{G}^{H}(x) .
$$

A more general statement is also easy to form, using exactly this. A general element in $\underline{A}(X)$ is a $G$-set over $X: f: Y \rightarrow X$. The above observation shows us how to pair $\underline{A}(X)$ and $\underline{M}(X)$ :

$$
f \otimes x \mapsto f_{*} f^{*}(x) .
$$

Understanding this action will help us make several computations related to equivariant homotopy groups.

We have a few distinguished ideals in the Burnside ring. First, note that if a $G$-set is induced, then any $G$-set we get by pull-back is also induced. This says that there is a "transfer" ideal in the Burnside ring. We can push slightly farther, though. If $\mathcal{F}$ is a family of subgroups of $G$, then we can form an ideal in the Burnside ring associated to $\mathcal{F}, I_{\mathcal{F}}$ as follows: begin with the ideal of $A(G)$ generated by $[G / H]$ for all $H \in \overline{\mathcal{F}}$, and then close up under restrictions and transfers. Put another way, this is the smallest ideal in $\underline{A}$ such that $\underline{I_{\mathcal{F}}}(G / H)=A(H)$ whenever $H \in \mathcal{F}$.

We shall see in $\S 4.3$ that this Mackey functor does occur as the homotopy groups of a relatively simple $G$-spectrum, namely the cofiber of various restriction maps. If $H_{i}$ runs through all proper subgroups of $G$, then the quotient Mackey functor has

$$
\underline{A / I}(G / H)= \begin{cases}\mathbb{Z} & H=G, \\ 0 & \text { otherwise. }\end{cases}
$$

This applies in several cases. For example, if $V$ is a representation such that $V^{H} \neq\{0\}$ for all proper subgroups $H$ but $V^{G}=\{0\}$, then $\pi_{0}\left(S^{V}\right)$ is this Mackey functor.

There is another key ideal which shows up, and the associated quotient is also named. Let $\underline{I}(G / H)$ denote the "kernel of the augmentation" $\underline{A}(G / H) \rightarrow \underline{A}(G /\{e\})=\mathbb{Z}$. This map takes a $G$-set $X$ to its cardinality. Since the map is the restriction, these kernels restrict to each other, and the condition that the transfer is a module map ensures that they are closed under transfers, and therefore form a Mackey functor. 
Definition 4.5. The augmentation ideal is the Mackey functor ideal $\underline{I}$ given by the kernel of the augmentation map.

The constant Mackey functor $\underline{\mathbb{Z}}$ is the quotient $\underline{A} / \underline{I}$. The value at $G / H$ is $\mathbb{Z}$ and all restriction maps are isomorphisms.

The Mackey functor $\underline{\mathbb{Z}}$ will play a central role in this paper, as computations with it are especially doable (unlike, say, with a more general Mackey functor). We will soon see how to compute the homology of any representation sphere $S^{V}$ with coefficients in $\underline{\mathbb{Z}}$.

4.2. $R O(G)$-Graded homotopy groups. One of the advantages of an honest equivariant setting is the presence of a large class of invertible spheres. If $W$ is a virtual representation of $G$, then we have a sphere $S^{W}$, and the assignment $W \mapsto S^{W}$ is monoidal where in the target we take the smash product. This gives us a large collection of homotopy groups.

Definition 4.6. The $R O(G)$-graded homotopy groups of $X$, denoted $\pi_{\star}(X)$, are given by

$$
\left[S^{W}, X\right], \quad W \in R O(G) .
$$

Just as before we can prolong this to a Mackey functor:

$$
\underline{\pi_{V}(X)}(G / H)=\left[G / H_{+} \wedge S^{V}, X\right] .
$$

This approach was pioneered by Lewis who used it to compute the $R O(G)$-graded homology of projective spaces $[\mathbf{2 1}]$. We will use this extensively to determine the homotopy groups of our spectrum $\tilde{\Omega}$.

The representation ring has a distinguished sub semiring: isomorphisms classes of genuine representations. We shall call this the "positive" part of the representation ring. Similarly, any virtual representation of the form $-V$ will be called "negative". Since the positive and the negative parts of the representation ring are sub-semirings, if $E$ is an equivariant ring spectrum, $\pi_{\star \geq 0} E$ and $\pi_{\star \leq 0} E$ are rings graded on honest representations. It is often easiest to give generators and relations for these, rather than $\pi_{\star} E$.

We can actually refine this approach slightly. The representation ring itself is a Mackey functor, and we can extend the assignment $W \mapsto S^{W}$ to a kind of Mackey functor in spaces.

Definition 4.7. If $W$ is a virtual representation of $H \subset G$, let

$$
\underline{\pi_{W}(X)}(G / K)=\left[G / K_{+} \wedge\left(G_{+} \wedge H S^{W}\right), X\right]_{G} .
$$

This will show up only obliquely in our proof. When we look at the cellular decomposition of $M U_{\left(C_{2^{n}}\right)}$, we will see extra classes exactly of this form. Having the additional homotopy groups would allow a cleaner description of the homology of $M U_{\left(C_{2^{n}}\right)}$. Additionally, this extra structure underscores the existence of additional restrictions and transfer maps. These transfer maps (especially related to the sign representations of subgroups of $C_{2^{n}}$ ) play a large role in determining differentials on normed classes. 
4.3. Some equivariant homotopy groups. Though the equivariant homotopy groups of spheres are monstrously difficult to compute, there are several cases where we can do so: we can compute $\pi_{0}\left(S^{V}\right)$ for any representation $V$. We begin with two observations which give the full form. The first is essentially a restatement of tom Deick's computations.

OBSERVATION 4.8. The abelian group $\pi_{0}^{G} S^{0}$ is the free abelian group generated by $[G / H]$ where $H$ ranges over all subgroups of $G$. The element $[G / H]$ is the image of 1 under the transfer map

$$
\pi_{0}^{H} S^{0}=A(H) \rightarrow A(G)=\pi_{0}^{G} S^{0} .
$$

for more general representations, we look at fixed points.

OBSERVATION 4.9. If $H$ is a subgroup of $G$ for which $V^{H} \neq\{0\}$, then

$$
\pi_{0}^{H^{\prime}} S^{V}=0
$$

for any $H^{\prime} \subset H$.

This is simply because to understand $\pi_{0} S^{0}\left(G / H^{\prime}\right)$, it suffices to restrict attention to $H^{\prime}$-Spectra and compute the Mackey functor $\pi_{0}$ there. Since we have a trivial summand, the computation reduces to that of $\pi_{0} S^{1}$.

The condition $V^{H} \neq\{0\}$ is clearly closed under subgroups of $H$ and under conjugation. Thus for any representation $V$, we have a family $\mathcal{F}_{V}$ : the subgroups of $G$ with non-trivial fixed points. The previous observations show the following result.

Proposition 4.10. As $\underline{A}$-modules,

$$
\underline{\pi_{0} S^{V}} \cong \underline{A} / \underline{I_{\mathcal{F}_{V}}} .
$$

There is a distinguished generator of $\underline{\pi_{0} S^{V}}$.

Definition 4.11. Let $a_{V}$ denote the inclusion of $S^{0}=\{0, \infty\}$ into $S^{V}$.

This class is a kind of Euler class [13]. Multiplication by $a_{V}$ gives us a map $\underline{A} \rightarrow \pi_{0} S^{V}$, and this realizes the above isomorphism. The following proposition is immediate from either the definition or our results about $\pi_{0} S^{V}$, but it will help us greatly in manipulating classes.

Proposition 4.12.

(1) If 1 denotes $\mathbb{R}$ with the trivial action, then $a_{1}=0$.

(2) For any $V$ and $W, a_{V \oplus W}=a_{V} \cdot a_{W}$.

(3) If $H \subset G$, then $\operatorname{Res}_{G}^{H}\left(a_{V}\right)=a_{\operatorname{Res}_{H} V}$.

(4) If $H \subset G$, then $N_{H}^{G}\left(a_{V}\right)=a_{I n d_{H}^{G} V}$.

In particular, we see that it suffices to consider $a_{V}$ where $V$ ranges over irreducible representations (though it will be notationally simpler to consider all such classes).

These classes are actually torsion-free in the homotopy groups of spheres. Their image in $\pi_{\star} H \underline{\mathbb{Z}}$, which we will also denote by $a_{V}$ is in general not. We can spell this out more explicitly. 
Proposition 4.13. If $\operatorname{Res}_{G}^{H}\left(a_{V}\right)=0$, then $|G / H| \cdot a_{V}=0$ in $\pi_{0} S^{V} \wedge H \underline{\mathbb{Z}}$.

Proof. The Hurewicz map on $\pi_{0}$ is the reduction modulo the augmentation ideal in the Burnside ring. In this ideal we have the obvious element $[G / H]-|G / H|[G / G]$.

For cyclic 2-groups (and more generally for cyclic $p$-groups), these classes have a very close connection to geometric fixed points and to the various families of subgroups one can consider.

TheOrem 4.14. For any $X$, we have

$$
\pi_{\star}\left(\tilde{E} \mathcal{F}_{m} \wedge X\right)=a_{\lambda\left(p^{m-1}\right)}^{-1} \pi_{\star}(X) .
$$

Proof. Since $\tilde{E} \mathcal{F}_{m}$ is the cofiber of the natural map $E \mathcal{F}_{m+} \rightarrow S^{0}$, we can identify it with

$$
\lim _{\rightarrow} S^{k \lambda\left(p^{m-1}\right)}
$$

where the maps in the colimit are the suspensions of $a_{\lambda\left(p^{m-1}\right)}$. The result follows.

Corollary 4.15. The homotopy groups of $\Phi^{G}(X)$ are given by the integer graded part of $a_{\sigma}^{-1} \pi_{\star}(X)$.

As was remarked above, the space $E \mathcal{F}_{m}$ is actually just a homotopy type. We could use any family of representations that had this property. In particular, we could add any collection of representations with a smaller stabilizer subgroup (named those with a smaller power of $\lambda$ ).

COROLlaRY 4.16. If we invert $a_{\lambda\left(p^{m-1}\right)}$ then we invert $a_{\lambda\left(p^{k}\right)}$ for all $k \leq m-1$.

COROLlaRY 4.17. Inverting $a_{\sigma}$ inverts $a_{V}$ for any representation $V$ with trivial fixed points.

This will make computations in the slice tower much simpler. In particular, we can use this to greatly reduce the complexity of the computations involving differentials in the slice tower.

\section{The slice tower and the slices of $M U_{\left(C_{2} n\right)}$}

5.1. The homology of $M U_{\left(C_{2^{n}}\right)}$. We begin with the following $C_{2^{-}}$ equivariant generalization of the classical splitting of $M U \wedge M U$ as $M U$ module spectra.

Proposition 5.1. As $M U_{\mathbb{R}}$-modules, we have a splitting

$$
M U_{\mathbb{R}} \wedge M U_{\mathbb{R}} \simeq M U_{\mathbb{R}} \wedge \bigvee_{p} S^{\frac{|p|}{2} \rho_{2}},
$$

where $p$ ranges over all monic monomials in $M U_{*}$. 
Since it will show up a very great deal, we give names to particular wedges of representation spheres.

DEFINITION 5.2. Let

$$
W=\bigvee_{p} S^{\frac{|p|}{2} \rho_{2}}
$$

where $p$ ranges over all monic monomials in $M U_{*}$.

The previous proposition gives us two immediate corollaries. First, it allows us to conclude the $R O(G)$-graded homology of $M U_{\mathbb{R}}$.

Corollary 5.3. We have a splitting

$$
H \underline{\mathbb{Z}} \wedge M U_{\mathbb{R}} \simeq H \underline{\mathbb{Z}} \wedge W .
$$

This can actually be shown geometrically using a Schubert cell decomposition of the Grassman manifolds which occur as the spaces in the $M U_{\mathbb{R}^{-}}$ spectrum. This splitting underscores also the importance of the regular representation sphere for $M U_{\mathbb{R}}$, and in our construction of the slice tower, we will capitalize on this.

The second corollary allows us to increase the size of the group.

Corollary 5.4. We have a splitting of $M U_{\left.\left(C_{2}\right)\right)}$-module spectra

$$
M U_{\left(C_{2^{n}}\right)} \wedge M U_{\left(C_{2^{n}}\right)} \simeq M U_{\left(C_{2^{n}}\right)} \wedge N_{C_{2}}^{C_{2^{n}}} W .
$$

Our understanding of the norm of a wedge allows us to reformulate this last part into a more explicit form.

THEOREM 5.5. We have an equivariant decomposition

$$
N_{C_{2}}^{C_{2^{n}}} W=\bigvee_{p \in I} C_{2^{n}} / H_{p+} \wedge S^{\frac{|p|}{\left|H_{p}\right|} \rho_{H p}},
$$

where $I$ is the orbits of monomials in $\pi_{*}^{\{e\}} M U_{\left(C_{2} n\right)} \otimes \mathbb{Z} / 2$ and where $H_{p}$ is the stabilizer subgroup of $p$.

REMARK 5.6. Since we are working modulo 2, it is immediate that $C_{2} \subset$ $H_{p}$ for all $p$. Thus we never have free summands in this wedge.

This in particular gives us a splitting of $H \underline{\mathbb{Z}} \wedge M U_{\left(C_{2^{n}}\right)}$, and we see that here the important cells are those of the form

$$
C_{2^{n+1}} / H_{+} \wedge S^{k \rho_{H}}
$$

for various non-trivial subgroups $H$. This slice tower capitalizes on this. By judiciously choosing an equivariant filtration, we can ensure that the associated graded of $M U_{\left(C_{2^{n}}\right)}$ is exactly $H \underline{\mathbb{Z}} \wedge M U_{\left(C_{2^{n}}\right)}$. As we shall see in the next section, this is very computable. 
5.2. Slice filtration. A key step in our solution is the production of a new equivariant filtration, the slice filtration. This is a refinement of the non-equivariant Postnikov tower, but it groups the homotopy according the regular representation cells. In fact, there is a choice here of "slice" cells, and different choices produce different slice spectral sequences. The choice we use plays nicely with the spectra $M U_{\left(C_{2^{n}}\right)}$, allowing us to identify the slice associated graded and to use it to compute equivariant homotopy groups.

Definition 5.7. Any generalized representation sphere of the form $G_{+} \wedge_{H} S^{k \rho_{H}-\epsilon}$, where $\epsilon$ is 0 or 1 , is a slice cell. The dimension of the slice cell $G_{+} \wedge_{H} S^{k \rho_{H}-\epsilon}$ is $k|H|-\epsilon$.

The inclusion of the factor $\epsilon$ seems initially somewhat confusing. This is actually also dictated by the Schubert cell decomposition of $M U_{\left(C_{2} n\right)}$. We know that in homology we see cells of the desired form (without any $\epsilon$ factor), but this means that they arise as cofibers of maps from a single desuspension. Having these desuspensions allows us to also control these maps, and not just the cells.

Associated to these collections of cells, we can build localizing subcategories.

Definition 5.8. Let $\mathcal{T}_{n}$ denote the full subcategory of $G$-spectra built by closing the category of all slice cells of dimension greater than $n$ under colimits and extensions.

In other words, we consider all spectra we can build out of the slice cells of dimension at least $n$ under iterated colimits and under extensions. We do not allow require (nor do we want to allow) arbitrary limits or fibers. This is a big difference between our slice filtration and the motivic one due to Voevodsky: the motivic slice filtration is a filtration of triangulated subcategories whereas ours in not.

Definition 5.9. The $n^{\text {th }}$ slice truncation functor $P^{n}$ is the Dror-nullification functor associated to the subcategory $\mathcal{T}_{n}$.

This is most easy described via its universal properties. We have $P^{n}$ and a natural transformation $1 \rightarrow P^{n}$ such that the space of maps $\operatorname{Map}(W$, $\left.P^{n}(X)\right)$ is equivariantly contractible for any slice cell $W$ of dimension at least $n$ and such that $P^{n}(X)$ is initial amongst spectra with this property [12].

It is easy to check that $G_{+} \wedge_{H} S^{k \rho_{H}-\epsilon}$ is $(k-\epsilon)$-connected if $k \geq 0$ and $(k|H|)$-connected if $k<0$. This give us a coarse lower bound on the connectivity of any element in $\mathcal{T}_{n}: n /|G|-1$. A general property of nullification then ensures the following fact (which for easy, we state only for $n>0$ ).

Proposition 5.10. The map $X \rightarrow P^{n}(X)$ is $(n /|G|-1)$-connected.

Dually, it is easy to show that for all $H, G_{+} \wedge_{H} S^{n} \in \mathcal{T}_{n}$. These are exactly the cells which are killed to build the equivariant Postnikov tower, we conclude the following. 
Proposition 5.11. For all $n$ and for all $k>n$,

$$
\underline{\pi_{k}\left(P^{n}(X)\right)}=0 \text {. }
$$

Definition 5.12. The slice tower of $X$ is the iterated nullification tower

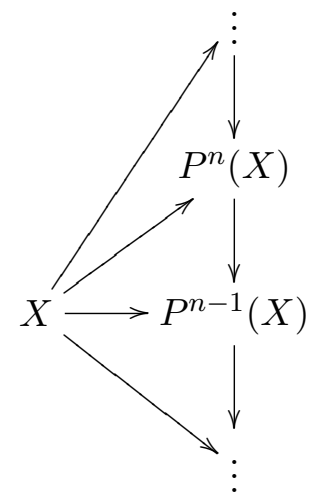

The slice spectral sequence is the spectral sequence associated to this filtration.

Proposition 5.10 ensures that the map from $X$ to the limit of the slice tower is a weak equivalence. Proposition 5.11 ensures that the colimit of the slice tower is contractible. Thus the slice spectral sequence has good convergence properties.

The $E_{1}$-term of this spectral sequence is given by the Mackey functor homotopy groups of the fibers $P^{n}(X) \rightarrow P^{n-1}(X)$. These fibers are the slices of $X$.

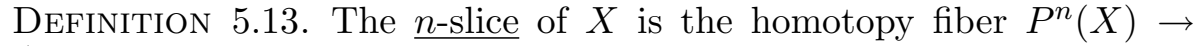
$P^{n-1}(X)$.

In general, determining the slices of $X$ is regrettably difficult. There are two cases where we can do so.

Proposition 5.14. If $T$ is a slice cell, then $T \wedge H \underline{\mathbb{Z}}$ is a $\operatorname{dim}(T)$-slice.

This is an essentially immediately computation of equivariant homology groups, and we postpone it until a later section.

Corollary 5.15. If again

$$
W=\bigvee_{p} S^{\frac{|p|}{2} \rho_{2}},
$$

then

$$
W \wedge H \underline{\mathbb{Z}} \quad \text { and } \quad\left(N_{C_{2}}^{C_{2} n} W\right) \wedge H \underline{\mathbb{Z}}
$$

are wedges of slices.

Proposition 5.16. The zero slice of $S^{0}$ is $H \underline{\mathbb{Z}}$. 
Proof. We prove this for cyclic $p$-groups, leaving the full case to the larger paper. The proof is by induction on the order of the group. We know that the slice tower is a refinement of the Postnikov tower. In particular, for the zero-slice, this means two things. First, the zero-slice of $S^{0}$ is of the form $H \underline{M}$ for some $\underline{M}$ and second, the underlying spectrum must be $H \mathbb{Z}$. In particular, though transfers of the element $1 \in \underline{M}(G /\{e\})$ might be killed, nothing that restricts to it can be.

We will show by induction on the group that any element in the augmentation ideal $I(G / G)$ can be realized by a map from some wedge of slice cells. The case for $G / H$ is handled identically. It suffices, by induction for transfer reasons, to then only consider elements in the augmentation ideal that are not in the image of the transfer from any subgroup. For a general group, these are generated by classes $[G / H]-|G / H|$ for $H$ a maximal proper subgroup of $G$. In the case of a cyclic group of prime power order, these are especially simple: there is only one such $H$ that is also a cyclic group of prime power order.

Since the restriction of $\alpha=\left[C_{p^{n}} / C_{p^{n-1}}\right]-p$ to $C_{p^{n-1}}$ is zero, we know that the composition

$$
C_{p^{n}+} \wedge_{C_{p^{n-1}}} S^{0} \rightarrow S^{0} \stackrel{\alpha}{\rightarrow} H \underline{A}
$$

is null-homotopic. Our understanding of how to build the a cell structure for $S^{\rho-1}$ shows that the 1-cells are attached by exactly the map

$$
C_{p^{n}+} \wedge_{p_{p^{n-1}}} S^{0} \rightarrow S^{0},
$$

and so $\alpha$ extends over the 1-skeleton. Since $H \underline{A}$ is an Eilenberg-Mac Lane spectrum, we conclude that $\alpha$ extends over $S^{\rho-1}$.

A similar proof actually shows that the category of zero slices is the category of Mackey functors for which all restriction maps are monomorphisms. This is true for an arbitrary finite group.

Proposition 5.17. Let $V$ be a representation, and let $\mathcal{F}_{V}$ be the family of subgroups $H$ of $G$ such that $V^{H} \neq 0$. Then for any Mackey functor $\underline{M}$ we have

$$
\left[S^{V}, H \underline{M}\right]=\left\{x \in \underline{M}(G / G) \mid \operatorname{Res}_{G}^{H}(x)=0, \forall H \in \mathcal{F}_{V}\right\} .
$$

Proof. Since $H \underline{M}$ is an Eilenberg-Mac Lane spectrum, we know

$$
\left[S^{V}, H \underline{M}\right]=\left[H \underline{\pi_{0} S^{V}}, H \underline{M}\right]=H o m_{\underline{A}}\left(\underline{\pi_{0} S^{V}}, \underline{M}\right) .
$$

The result now follows from our earlier analysis of $\pi_{0} S^{V}$.

For our purposes, the most important example where we can determine the slice associated graded is $M U_{\left(C_{2^{n}}\right)}$. 
Theorem 5.18 (the Slice Theorem). There is an equivariant filtration of $M U_{\left(C_{2} n\right)}$ (the slice filtration) such that the associated graded is

$$
H \underline{\mathbb{Z}} \wedge \bigvee_{p \in I} C_{2^{n}} / H_{p+} \wedge S^{\frac{|i|}{\left|H_{p}\right|} \rho_{H_{p}}},
$$

where $I$ is the orbits of monomials in $\pi_{*}^{\{e\}} M U_{\left(C_{2} n\right)} \otimes \mathbb{Z} / 2$ and where $H_{p}$ is the stabilizer subgroup of $p$.

Rather than give a complete proof, we will sketch the argument. We begin with a statement about the underlying homotopy of $M U_{\left(C_{2^{n}}\right)}$.

Proposition 5.19. We can find generators of $\pi_{*}^{\{e\}} M U_{\left(C_{2^{n}}\right)}$ such that as an equivariant algebra

$$
\pi_{*}^{\{e\}} M U_{\left(C_{2^{n}}\right)}=\mathbb{Z}\left[r_{1}, \ldots, \gamma^{2^{n-1}-1} r_{1}, r_{2}, \ldots\right],
$$

where $\gamma$, the generator of $C_{2^{n}}$, acts in the obvious way, and where $\gamma^{2^{n-1}} r_{i}=$ $(-1)^{i} r_{i}$. The degree of $r_{i}$ is $2 i$.

For $n=1$, these can be chosen to be the usual classes. For $n=2$, we can choose these to (up to decomposables) be the usual classes $t_{i}$.

These underlying homotopy classes are actually the restriction of $C_{2}$-equivariant maps from non-trivial representation spheres, and from this we get a good description of equivariant generators in general.

Proposition 5.20. There are $C_{2}$-equivariant maps

$$
\bar{r}_{i}: S^{i \rho_{2}} \rightarrow M U_{\left(C_{2^{n}}\right)}
$$

such that the underlying map is $r_{i}$.

When we need to remember the ambient group, we will further adorn $\bar{r}_{i}$ with a second subscript: $\bar{r}_{i, n}$ is $\bar{r}_{i}$ for $C_{2^{n}}$ and $M U_{\left(C_{\left.2^{n}\right)}\right.}$. The left unit allows us to find $\bar{r}_{i, k}$ in $\pi_{i \rho_{2}} M U_{\left(C_{2^{n}}\right)}$ for all $k \leq n$.

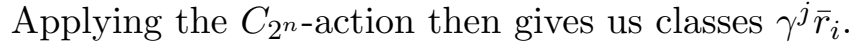

Definition 5.21. Let $S\left[\bar{r}_{i}\right]$ denote the free $A_{\infty}$-ring spectrum on $S^{i \rho_{2}}$.

Thus

$$
S\left[\bar{r}_{i}\right]=\bigvee_{j \geq 0} S^{i j \rho_{2}},
$$

and a map of associative algebras $S\left[\bar{r}_{i}\right] \rightarrow X$ is given by an element $\pi_{i \rho_{2}} X$. As the name implies, there is for each $i$ a distinguished map $S\left[\bar{r}_{i}\right] \rightarrow$ $M U_{\left(C_{2^{n}}\right)}$. Since $M U_{\left(C_{2^{n}}\right)}$ is a commutative ring spectrum, we can smash all of these algebras together and get a map of associative algebras

$$
\bigwedge_{k \geq 1} S\left[\bar{r}_{k}\right] \rightarrow M U_{\left(C_{2^{n}}\right)}
$$


The underlying map for this exactly picks out the subalgebra generated by the generators $r_{k}$ in homotopy.

If we apply the norm, followed by the canonical map $N_{C_{2}}^{C_{2} n} M U_{\left(C_{2} n\right)} \rightarrow$ $M U_{\left(C_{2^{n}}\right)}$, then we get a map of associative algebras

$$
N\left(\bigwedge_{k \geq 1} S\left[\bar{r}_{k}\right]\right) \rightarrow M U_{\left(C_{2^{n}}\right)}
$$

Let $R$ denote the associative algebra $N\left(\bigwedge_{k>1} S\left[\bar{r}_{k}\right]\right)$.

Since $S\left[\bar{r}_{k}\right]$ is a wedge of equivariant spheres, we can easily understand

$$
R=\bigwedge_{k \geq 1} N\left(S\left[\bar{r}_{k}\right]\right) .
$$

Proposition 5.22. The homology of the underlying spectrum for $R$ is

$$
\mathbb{Z}\left[r_{1}, \ldots, \gamma^{2^{n-1}-1} r_{1}, r_{2}, \ldots\right],
$$

where $r_{i}$ is the fundamental class of the associated sphere, and where $\left|r_{i}\right|=$ $2 i$, and the underlying map on homotopy

$$
\pi_{*}^{\{e\}} R \rightarrow \pi_{*}^{\{e\}} M U_{\left(C_{2^{n}}\right)}
$$

is surjective, sending fundamental classes to the identically named elements.

In the group action, $\gamma^{2^{n-1}}$ acts as $(-1)^{i}$ on $r_{i}$. In fact, this sign action is recorded in the equivariant homotopy by the fact that the conjugation map $S^{\rho_{2}} \rightarrow S^{\rho_{2}}$ had degree -1 . We also know that every class in the norm of our wedge has a stabilizer group containing $C_{2}$. Putting these two facts together leads us to consider orbits of monomials in the underlying homotopy of $M U_{\left(C_{2} n\right)}$. Associated to each orbit, we can attach the stabilizer subgroup modulo 2, stripping out the pesky sign problem. For easy of notation, if $p$ is a monomial, let $H_{p}$ denote this stabilizer subgroup.

This gives us exactly the equivariant homotopy type of $R$ : it is a wedge of induced spheres of the form

$$
C_{2^{n}+\wedge_{H_{p}}} S^{\frac{|p|}{\left|H_{p}\right|} \rho_{H_{p}}}
$$

where $p$ ranges over a set of representatives for the orbits of monomials in the underlying homotopy of $M U_{\left(C_{2^{n}}\right)}$.

The reader will at this point no doubt recognize that the claim of the necessary theorem is that $M U_{\left(C_{2} n\right)}$ has an equivariant filtration for which the associated graded is $H \underline{\mathbb{Z}} \wedge R$. We can achieve this by first recognizing that since $R$ is essentially a polynomial algebra in spectra (though we stress that the variables do not commute with themselves in the sense that there are no power operations beyond the $p^{\text {th }}$ power), we have a filtration by degree, with the "degree at least $n$ " bimodule being denoted $M_{n}$. Using the map of associative algebras $R \rightarrow M U_{\left(C_{2^{n}}\right)}$, we get a decreasing filtration of 
$M U_{\left(C_{\left.2^{n}\right)}\right.}=M U_{\left(C_{2^{n}}\right)} \wedge_{R} R$ by considering $M U_{\left(C_{2^{n}}\right)} \wedge_{R} M_{n}$. The associated graded for this filtration is simply

$$
\left(M U_{\left(C_{2} n\right)} \wedge_{R} S^{0}\right) \wedge R
$$

This gives rise to by far the most difficult theorem in our proof: the Reduction Theorem. Since the proof is somewhat technical, we will not reproduce it here.

Theorem 5.23 (the Reduction Theorem). We have an equivariant equivalence

$$
M U_{\left(C_{2^{n}}\right)} \wedge_{R} S^{0} \simeq H \underline{\mathbb{Z}} .
$$

In fact, the map $M U_{\left(C_{2^{n}}\right)} \wedge_{R} S^{0} \rightarrow H \underline{\underline{Z}}$ realizing this equivalence is the 0th Postnikov section of $M U_{\left(C_{2}{ }^{n}\right)}$. We should interpret the Reduction Theorem as an equivariant analogue of the fact that $M U$ modulo all of its generators in non-zero degree is $H \mathbb{Z}$. As a consequence of the Reduction Theorem, we also see that $M U_{\left(C_{\left.2^{n}\right)}\right.} \wedge_{R} S^{0}$ inherits an algebra structure. This is not immediate, since $R$ is not commutative.

The astute reader will notice that at no point in the argument up to this point have we used the slice filtration. It is precisely in our proof of the reduction theorem that having the full slice story becomes most useful.

With the Reduction Theorem, the description of the associated graded of $M U_{\left(C_{2} n\right.}$ is complete. Before focusing on the specific computations, we give a brief corollary.

COROllary 5.24. For any $k$, there is an equivariant filtration of $\Sigma^{k \rho_{2^{n}}} M U_{\left(C_{2^{n}}\right)}$ such that the associated graded is

$$
H \underline{\mathbb{Z}} \wedge \bigvee_{p \in I} C_{2^{n}} / H p+\wedge S^{\frac{|i|+k 2^{n}}{\left|H_{p}\right|} \rho_{H_{p}}},
$$

where again $I$ is the orbits of monomials in $\pi_{*}^{\{e\}} M U_{\left(C_{2^{n}}\right)} \otimes \mathbb{Z} / 2$ and where $H_{p}$ is the stabilizer subgroup of $p$.

This is immediate from smashing the filtration of $M U_{\left(C_{2} n\right)}$ with $S^{k \rho_{2^{n}}}$. Here we have used that the restriction of the regular representation of $C_{2^{n}}$ to any subgroup is a sum of copies of the regular representation. In particular, we see that the form of the summands,

$$
H \underline{\mathbb{Z}} \wedge\left(C_{2^{n}}+\wedge_{C_{2^{k}}} S^{j \rho_{2^{k}}}\right),
$$

is unchanged!

\section{The homology of a point, and the Gap theorem}

For cyclic groups of prime-power order and for a virtual representation spheres $S^{V}$, it is easy to compute the Mackey functor homotopy group $\pi_{*}\left(S^{V} \wedge H \underline{Z}\right)$. Using the linear ordering of the subgroups, we can build a triangularization of $S^{V}$ which gives us an especially simple chain complex. 
For ease of exposition, we will restrict attention to cyclic 2-groups. Everything said here applies equally well to cyclic $p$-groups. Our exposition is greatly streamlined by the following obvious fact: the subgroups of a cyclic $p$-group are linearly ordered.

The analysis begins with a decomposition of $S^{V}$ into cells for $V$ an honest representation. For this, we can actually understand this in spaces. This gives us, by equivariant Spanier-Whitehead duality, a cellular decomposition of $S^{-V}$, and then by smashing, we get one for any virtual representation. This translates into our chain complexes by simply requiring us to form the associated cochain complex for $S^{-V}$ and then to tensor things together for a more general case. We remark that for honest representations, this chain complex is much smaller than the one you get by decomposing $V$ into irreducible representations and tensoring the associated complexes together.

For the rest of this section, let $V$ be a fixed representation of $C_{2^{n}}$.

6.1. The Bredon homology of $S^{V}$. We can decompose $V$ into a direct sum of representations with a fixed stabilizer subgroup:

$$
V=\bigoplus_{k=0}^{n} V_{k}
$$

where $V_{k}$ is a sum of representations of the form $\lambda(j)$ with the 2-adic valuation of $j$ equal to $n-k$. Thus $V_{i}$ is stabilized by $C_{2^{n-i}}$, and conversely, the fixed points of $C_{2^{n-i}}$ is

$$
V^{C_{2}-i}=\bigoplus_{k=0}^{i} V_{k}
$$

This actually immediately lets us built a cellular decomposition. Since any representation spheres $S^{\lambda(i)}$ and $S^{\lambda\left(i^{\prime}\right)}$ are equivalent if $i$ and $i^{\prime}$ have the same 2 -adic valuation, we can simple consider

$$
V=m_{0} \oplus m_{1} \sigma \oplus m_{2} \lambda\left(2^{n-2}\right) \oplus \cdots \oplus m_{n} \lambda .
$$

This particular ordered decomposition of $V$ into irreducible representations with shrinking stabilizer groups gives our cells. For ease on the reader's eyes, let

$$
M_{k}=\operatorname{dim} V^{C_{2^{n-k}}}=m_{0}+m_{1}+2 m_{2}+\cdots+2 m_{k} .
$$

Proposition 6.1. There is a cell decomposition of $S^{V}$ of the form

$$
S^{m_{0}} \cup \bigcup_{j_{1}=1}^{m_{1}} C_{2^{n}}+\wedge_{C_{2^{n-1}}} e^{M_{0}+j_{1}} \cup \cdots \cup \bigcup_{j_{n}=1}^{2 m_{n}} C_{2^{n}}+\wedge e^{M_{n-1}+j_{n}} .
$$

Moreover, the subcomplexes

$$
S^{m_{0}} \cup \bigcup_{j_{1}=1}^{k} C_{2^{n}+} \wedge_{C_{2^{n-1}}} e^{m_{0}+j_{1}}
$$


and

$$
S^{m_{0}} \cup \bigcup_{j_{1}=1}^{m_{1}} C_{2^{n}+} \wedge_{C_{2^{n-1}}} e^{m_{0}+j_{1}} \cup \cdots \cup \bigcup_{j_{k}=1}^{2 \ell} C_{2^{n}+} \wedge_{C_{2^{n-k}}} e^{M_{k-1}+j_{k}} .
$$

are the representation spheres

$$
S^{m_{0}+k \sigma} \text { and } S^{m_{0}+m_{1} \sigma+\cdots+\ell \lambda\left(2^{n-k}\right)}
$$

respectively.

The second half essentially tells us how to build these. We attach cells with increasingly smaller stabilizer groups to the representation spheres we have already built. We can therefore understand the cell structure by understanding how to build $S^{V \oplus \lambda\left(2^{k}\right)}$ with $S^{V}$ as the co-dimension 2-skeleton for representations $V$ with stabilizer containing $C_{2^{k}}$.

This is easiest to visualize with a picture. In Figure 3, the $z$-axis represents the representation $V$, while the $(x, y)$-plane (herein depicted as $\left.\lambda\left(2^{n-2}\right)\right)$ is $\lambda\left(2^{k}\right)$. The white dot in the center is the origin, and the shading on the semi-planes indicates the contribution of the group action on the vertical component (in this case, flipping back and forth).

We have a natural cell structure for $S^{\lambda\left(2^{k}\right)}$ :

$$
S^{0} \cup C_{2^{n}}+\wedge_{C_{2^{n-k}}} e^{1} \cup C_{2^{n}}+\wedge_{C_{2^{n-k}}} e^{2} .
$$

We can easily visualize this cell structure on the Riemann sphere. The 0-cells are the origin and the point at infinity, the 1-cells are the great semi-circles through the zero cells and the $2^{k \text { th }}$-roots of unity, and the 2 -cells interpolate between these. This is depicted in Figure 4 for $k=3$.

To build our cell structure on $S^{V \oplus \lambda\left(2^{k}\right)}$, we smash this cell structure with the spheres already built. Since the stabilizer subgroup of $V$ contains $C_{2^{n-k}}$, we know that

$$
S^{V} \wedge\left(C_{2^{n}+} \wedge_{C_{2^{n-k}}} e^{1}\right)=C_{2^{n}+} \wedge_{C_{2^{n-k}}} S^{V} \wedge e^{1}=C_{2^{n}+} \wedge_{C_{2^{n-k}}} e^{1+\operatorname{dim} V},
$$

Thus we can ignore the codimension 1-skeleton of $S^{V}$ after smashing with $C_{2^{n}} / C_{2^{n-k}}$, and we produce the desired cell structure on $S^{V \oplus \lambda\left(2^{k}\right)}$.

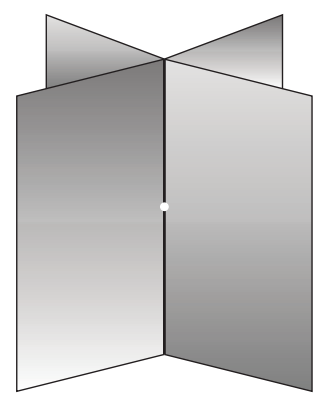

Figure 3. Adding New Cells 


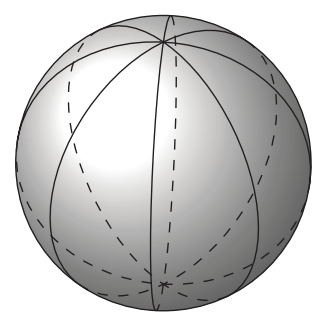

FiguRE 4

This gives us very simple chain complexes to compute the Bredon homology.

Definition 6.2. If $V=m_{0}+m_{1} \sigma+m_{2} \lambda\left(2^{n-2}\right)+\cdots+m_{n} \lambda$, then let $C(V)$ denote the chain complex

$$
C(V)_{j}= \begin{cases}\mathbb{Z}\left[C_{2^{n}} / C_{2^{n-k}}\right] & M_{k-1}<j \leq M_{k} \\ 0 & \text { otherwise }\end{cases}
$$

where the differentials are those equivariant maps such that the restriction of the complex to those cells of dimension at most $M_{k-1}+2 j$ is quasi-isomorphic to the chains on $S^{M_{k-1}+2 j}$.

The complex $C(V)$ is the equivariant cellular chain complex. If we take fixed points for various subgroups and take homology, then we recover the Bredon homology groups, by definition.

Proposition 6.3. As Mackey functors,

$$
\underline{H_{n}\left(S^{V}\right)}(G / H):=\underline{\pi_{n}\left(S^{V} \wedge H \underline{Z}\right)}(G / H)=H_{n}\left(C(V)^{H}\right) .
$$

We can rewrite the final step as saying that homotopy classes of equivariant maps from the chain complex that is $\mathbb{Z}[G / H]$ in dimension $n$ to $C(V)$ is the same as the homology of the fixed points of the complex.

The most important cases are regular representations spheres, and we spell out several cases for the reader's convenience. From these, the general pattern easily follows.

ExAmple 6.4 . For $C_{4}$, we have

$$
C\left(\rho_{4}\right)=\mathbb{Z}\left[C_{4} / C_{4}\right] \longleftarrow \nabla
$$

where $\nabla$ is the "fold" map $\nabla: \mathbb{Z}^{2} \rightarrow \mathbb{Z}$. We also have

$$
C\left(2 \rho_{4}\right)=\mathbb{Z} \longleftarrow \mathbb{Z}^{2} \stackrel{1-\gamma}{\longleftarrow} \mathbb{Z}^{2} \stackrel{1+\gamma}{\longleftarrow} \mathbb{Z}^{4} \stackrel{1-\gamma}{\longleftarrow} \mathbb{Z}^{4} \stackrel{T r}{\longleftarrow} \mathbb{Z}^{4} \stackrel{1-\gamma}{\longleftarrow} \mathbb{Z}^{4},
$$

where we have abreviated $\mathbb{Z}\left[C_{4} / H\right]$ to $\mathbb{Z}^{(4 /|H|)}$ and where $\operatorname{Tr}=(1+\gamma+$ $\left.\gamma^{2}+\gamma^{3}\right)$ 
There is an essential fact that underlies all of what follows. If $V$ contains $\sigma$, then $C(V)$ always begins

$$
\mathbb{Z}\left[C_{2^{n}} / C_{2^{n}}\right] \stackrel{\nabla}{\longleftarrow}\left[C_{2^{n}} / C_{2^{n-1}}\right]
$$

This quite simple result is the basis for the gap theorem!

6.2. The Bredon homology of $S^{-V}$. Since $S^{-V}$ is the equivariant Spanier-Whitehead dual of $S^{V}$, the Bredon chains are the dual to the chains on $S^{V}$ :

$$
C(-V):=\operatorname{Hom}(C(V), \mathbb{Z})
$$

where $\mathbb{Z}$ has the trivial action. The two most important examples are $C\left(\rho_{2^{n}}\right)$ and $C\left(2 \rho_{2^{n}}\right)$. We again illustrate these for $C_{4}$.

EXAMPLE 6.5. We have

$$
C\left(-\rho_{4}\right)=\mathbb{Z}\left[C_{4} / C_{4}\right] \rightarrow \mathbb{Z}\left[C_{4} / C_{2}\right] \rightarrow \mathbb{Z}\left[C_{4}\right] \rightarrow \mathbb{Z}\left[C_{4}\right],
$$

where now the first map is the diagonal $\mathbb{Z} \rightarrow \mathbb{Z}^{2}$, and the remaining ones are as in the positive case.

We also have

$$
\begin{aligned}
& C\left(-2 \rho_{4}\right)= \\
& \quad \mathbb{Z}\left[C_{4} / C_{4}\right] \rightarrow \mathbb{Z}\left[C_{4} / C_{2}\right] \rightarrow \mathbb{Z}\left[C_{4} / C_{2}\right] \rightarrow \mathbb{Z}\left[C_{4}\right] \rightarrow \mathbb{Z}\left[C_{4}\right] \rightarrow \mathbb{Z}\left[C_{4}\right] \rightarrow \mathbb{Z}\left[C_{4}\right],
\end{aligned}
$$

and again the only change is that the first map is the diagonal map.

Proposition 6.6. If $V$ contains a copy of $\sigma$, then the two non-zero terms of largest degree in $C(-V)$ are

$$
\mathbb{Z}\left[C_{2^{n}} / C_{2^{n}}\right] \stackrel{\Delta}{\longrightarrow} \mathbb{Z}\left[C_{2^{n}} / C_{2^{n-1}}\right],
$$

where $\Delta$ is the diagonal map $\mathbb{Z} \rightarrow \mathbb{Z}^{2}$.

6.3. The Gap theorem. We begin with a few simple homotopy groups.

Lemma 6.7. For any $k>0$ and any $j$, we have

$$
\pi_{-3,-2,-1}\left(H \underline{\mathbb{Z}} \wedge C_{2^{n}}+\wedge_{C_{2^{k}}} S^{j \rho_{2^{k}}}\right)=0 .
$$

PROOF. By the natural adjunctions between induction and restriction, it will suffice to show that for any $j$

$$
\pi_{-3,-2,-1} H \underline{\mathbb{Z}} \wedge S^{j \rho_{2^{n}}}=0 .
$$

If $j \geq 0$, then elementary connectivity arguments show this is so. For $j<$ -3 , the $(-j)$-connectivity of $S^{-j \rho_{2} n}$ produces the desired co-connectivity. For $j$ between -3 and -1 inclusive, Proposition 6.6 shows that in dimensions $j$ and $j-1, C\left(j \rho_{2^{n}}\right)$ looks like

$$
\mathbb{Z}\left[C_{2^{n}} / C_{2^{n}}\right] \stackrel{\Delta}{\rightarrow} \mathbb{Z}\left[C_{2^{n}} / C_{2^{n-1}}\right] .
$$


Upon passage to $C_{2^{n}}$ fixed points, this complex becomes

$$
\mathbb{Z} \stackrel{1}{\rightarrow} \mathbb{Z}
$$

and we conclude that in degrees $j$ and $j-1$, the homology is 0 . The only remaining case is $j=-1$ and degree -3 . It is easy to check that in this case, the map from degree -3 to -4 is injective, resulting in a trivial homology group in degree -3 .

As an immediate consequence of this and the Slice Theorem, we learn a quite surprising fact about the homotopy of the fixed points of $M U_{\left(C_{2^{n}}\right)}$.

TheOREM 6.8. For any $k$,

$$
\pi_{-3,-2,-1} \Sigma^{k \rho_{2} n} M U_{\left(C_{2^{n}}\right)}=0 .
$$

Proof. For $k \geq 0$, this is immediate, since $M U_{\left(C_{2} n\right)}$ is $(-1)$-connected, and $S^{k \rho_{2} n}$ is $k$-connected. For $k<0$, we use Corollary 5.24. Associated to this filtration, we have a spectral sequence computing equivariant homotopy. The $E_{1}$-term is the equivariant homotopy of terms of the form

$$
H \underline{\mathbb{Z}} \wedge C_{2^{n}}+\wedge_{C_{2} m} S^{j \rho_{2} m}
$$

where $m>0$. By Lemma $6.7, \pi_{-3,-2,-1}$ of this is zero. Since $\pi_{-2}$ of every term in the associated graded is zero, we conclude the same is true for $\Sigma^{k \rho_{2^{n}}} M U_{\left(C_{2^{n}}\right)}$.

Theorem 6.9 (the Gap Theorem). If $\bar{\Delta}: S^{k \rho_{2^{n}}} \rightarrow M U_{\left(C_{2^{n}}\right)}$ is any equivariant homotopy class, then

$$
\pi_{-3,-2,-1} \bar{\Delta}^{-1} M U_{\left(C_{2^{n}}\right)}=0 .
$$

Proof. Inverting $\bar{\Delta}$ involves forming a directed colimit

$$
\cdots \rightarrow M U_{\left(C_{2^{n}}\right)} \stackrel{\bar{\Delta}}{\longrightarrow} \Sigma^{-k \rho_{2^{n}}} M U_{\left(C_{2^{n}}\right)} \stackrel{\bar{\Delta}}{\longrightarrow} \ldots
$$

Spheres are still compact objects, and so we conclude

$$
\pi_{-3,-2,-1} \bar{\Delta}^{-1} M U_{\left(C_{2^{n}}\right)}=\lim _{\rightarrow} \pi_{-3,-2,-1} \Sigma^{m k \rho_{2^{n}}} M U_{\left(C_{2^{n}}\right)}=\lim _{\rightarrow} 0=0 .
$$

Thus in a huge family of equivariantly periodic spectra (these with period $k \rho_{2^{n}}$ ), we always have a gap in the homotopy of the fixed points. This is actually quite shocking and strong. With very little work, we have produced one of the key steps in our theorem: the Kervaire classes are eventually detected in the zero group.

REMARK 6.10. There are two known antecedents to this result. Atiyah showed that the fixed points of $K_{\mathbb{R}}$ are $K O$, and the standard computation of the homotopy of $K O$ has $\pi_{-3,-2,-1} K O=0$. Similarly, unpublished work of Mahowald-Rezk and Goerss-Henn show that in the homotopy of $E O_{2}\left(C_{4}\right)=$ $E_{2}^{C_{4}}$, we have $\pi_{-3,-2,-1} E O_{2}\left(C_{4}\right)=0$. Our result shows that these vanishing results are actually quite generic. 


\section{Some cohomology operations and slice differentials}

The Periodicity and Homotopy Fixed Points Theorems rely on a simple cohomological computation. We construct several elements in the equivariant Steenrod algebra in $B P^{(n)}$-modules and we show that these act nontrivially on the powers of $u_{2 \sigma}$. Tying these to $N\left(\bar{r}_{i}\right)$ for various $i$ produces for us, by definition, slice differentials.

7.1. Naming elements in Bredon homology. One of the most useful features of this approach is that we can easily understand the full ring structure of $\pi_{\star} H \underline{\mathbb{Z}}$ (at least the positive part). In particular, we can explicitly name every element, and this allows us to better understand how elements in the slice spectral sequence interact. We saw that the earlier computations that if $V$ is an oriented representation (that is, the generator acts via a degree 1 map on the underlying sphere), then we have a distinguished $\operatorname{map} S^{\operatorname{dim}(V)} \rightarrow S^{V} \wedge H \underline{\mathbb{Z}}$.

Definition 7.1. Let $u_{V}$ denote the orientation map $S^{\operatorname{dim}(V)} \rightarrow S^{V} \wedge H \underline{\mathbb{Z}}$.

Just as with $a_{V}$, these satisfy a number of very useful properties.

Proposition 7.2.

(1) If 1 is the trivial representation, then $u_{1}=1$.

(2) If $V$ and $W$ are representation, then $u_{V \oplus W}=u_{V} \cdot u_{W}$.

(3) If $H \subset G$, then $N_{H}^{G}\left(u_{V}\right) \cdot u_{\operatorname{dim}(V) \operatorname{Ind}_{H}^{G} 1}=u_{\text {Ind } d_{H}^{G} V} \cdot$

The only non-obvious result is the third. This follows immediately from writing out the norm of the source.

This lets us name every element in our chain complexes. We give the generic example for $C_{8}$ and various copies of the regular representation thereof.

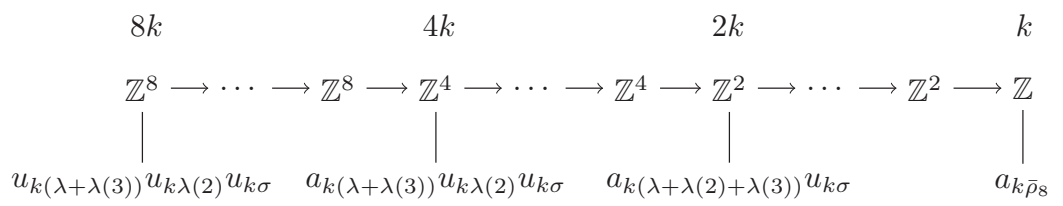

This method of naming actually also follows quite quickly from our choice of cell-structure. We restrict attention to orientable $V$ for now and will indicate the simple change for non-orientable $V$ at the end. Since the codimension 2-skeleton of our cell-structure is another representation sphere (and one for which the stabilizer subgroup is larger), it will suffice to understand how to name the elements of $S^{V+\lambda}$ relative to those of $S^{V}$. This follows from the following.

Observation 7.3. The inclusion $S^{V} \rightarrow S^{V+\lambda}$ is $a_{\lambda}$.

Thus for $* \leq \operatorname{dim}(V)$, multiplication by $a_{\lambda}$ gives an isomorphism

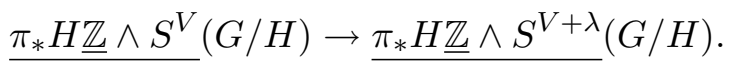


It is also immediate from the chain complexes that the $\operatorname{dim}(V)+1$-dimensional homology vanishes. Finally, by definition, the $\operatorname{dim}(V+\lambda)$-homology is generated by $u_{V+\lambda}$. Downward induction on the number of summands then names generators for all homology groups.

So what happens in the non-orientable case? The names here change very slightly. If $V$ is non-orientable, then $V$ contains an odd number of copies of $\sigma$, and $V-\sigma$ is orientable. We name the elements of the homology of $S^{V-\sigma}$ in the usual way, and composing with $a_{\sigma}$ produces the generators of the homology of $S^{V}$. In particular, we see that if $V$ is not orientable, then all homology groups of $S^{V}$ are 2-torsion.

Though not directly needed, we also list some multiplicative relations in the Bredon homology of a point. These will result is drastic simplifications upon inverting $a_{\sigma}$, which in turn will make our computation of several cohomology operations tractable.

Proposition 7.4. The elements $u_{\lambda\left(2^{k}\right)}$ are subject to the following relations

$$
2^{j} a_{\lambda\left(2^{i}\right)} u_{\lambda\left(2^{i+j}\right)}=a_{\lambda\left(2^{i+j}\right)} u_{\lambda\left(2^{i}\right)},
$$

where here we have used that $\lambda\left(2^{n-1}\right)=2 \sigma$.

It is then immediate that for all $k<n-1$,

$$
a_{\sigma}^{3} u_{\lambda\left(2^{k}\right)}=0
$$

since $a_{\sigma}^{2} u_{\lambda\left(2^{k}\right)}$ is divisible by 2 .

Though we have not described the other equivariant homology groups, it is not difficult to check that $a_{\sigma}$ also acts nilpotently on almost all classes there. More specifically, we have the following.

Proposition 7.5. As rings,

$$
a_{\sigma}^{-1} \pi_{\star} H \underline{\mathbb{Z}}=\mathbb{Z} / 2\left[a_{\sigma}^{ \pm 1}, a_{\lambda\left(2^{n-2}\right)}^{ \pm 1}, \ldots, a_{\lambda}^{ \pm 1}, u_{2 \sigma}\right],
$$

and as spectra, $\Phi^{G} H \underline{\mathbb{Z}}=H \mathbb{Z}[b]$, where $b=u_{2 \sigma} / a_{2 \sigma}$ is in degree 2 .

7.2. Relative cohomology operations. For sake of argument, we assume that $B P^{(n)}$ is an $E_{\infty}$ ring spectrum. If this is not the case, then we replace it with $M U_{\left(C_{2^{n}}\right)}$. While in general there are many more classes that will appear for $M U_{\left(C_{2}\right)}$, we will restrict attention to geometric fixed points (for which things are better behaved). We begin by analyzing the spectrum $H \underline{\mathbb{Z}} \wedge_{B P^{(n)}} H \underline{\mathbb{Z}}$. This is an equivariant $E_{\infty}$ ring spectrum whose homotopy is the ring of cooperations for homology in the category of $B P^{(n)}$-modules.

In general, the homotopy of this will be very difficult to compute. However, we can identify particular elements which we can easily check to be non-zero by mapping to the geometric fixed points. To begin, we quickly compute the geometric fixed points of this spectrum. 
Proposition 7.6. As commutative ring spectra,

$$
\Phi^{G}\left(H \underline{\mathbb{Z}} \wedge_{B P^{(n)}} H \underline{\mathbb{Z}}\right)=H \mathbb{F}_{2}\left[b^{\prime}\right] \wedge_{H \mathbb{F}_{2}} H \mathbb{F}_{2}[b]=H \mathbb{F}_{2}\left[b, b^{\prime}\right],
$$

where as before $b=b^{\prime}=u_{2 \sigma} / a_{2 \sigma}$ is an element in degree 2 .

Before continuing, we make a very brief digression into names of elements. Just as classically, the classes $\bar{r}_{i, n}$ do not occur in $B P^{(n)}$ unless $i$ is of the form $2^{j}-1$. We therefore give new $B P^{(n)}$-names to simplify the writing. Similarly, since in this section $n$ is held fixed, we suppress it from the notation.

DeFinition 7.7. Let $\bar{v}_{i}$ denote the element $\bar{r}_{2^{i}-1, n}$.

Even using $M U_{\left(C_{2^{n}}\right)}$, these are the elements that play a key role (since we always have a splitting in equivariant $A_{\infty}$-algebras that splits off $\left.B P^{(n)}\right)$.

We define our homology classes inductively using the cofiber sequences

$$
\left(G \cdot \bar{v}_{i}\right) B P^{(n)} \rightarrow B P^{(n)} \rightarrow B P^{(n)} /\left(G \cdot \bar{v}_{i}\right) .
$$

A key observation that makes many of the computations here work is that from the perspective of geometric fixed points, there is no difference between $\left(G \cdot \bar{v}_{i}\right) B P^{(n)}$ and $N\left(\bar{v}_{i}\right) B P^{(n)}=\Sigma^{i \rho_{G}} B P^{(n)}$, and the map to $B P^{(n)}$ is multiplication by $N\left(\bar{v}_{i}\right)$. This is because all other elements are carried by induced spheres and are therefore wiped out by the geometric fixed points construction.

If we smash this over $B P^{(n)}$ with $H \underline{\mathbb{Z}}$, then we again get a cofiber sequence:

$\left(G \cdot \bar{v}_{i}\right) B P^{(n)} \wedge_{B P^{(n)}} H \underline{\mathbb{Z}} \rightarrow B P^{(n)} \wedge_{B P^{(n)}} H \underline{\mathbb{Z}} \rightarrow B P^{(n)} /\left(G \cdot \bar{v}_{i}\right) \wedge_{B P^{(n)}} H \underline{\mathbb{Z}}$,

and from this we can identify various homotopy elements. Let $N\left(\bar{v}_{i}\right)$ denote the homotopy class $S^{\left(2^{i}-1\right) \rho_{G}} \rightarrow\left(G \cdot \bar{v}_{i}\right) B P^{(n)} \wedge_{B P^{(n)}} H \underline{Z}$ induced by the Thom reduction map $B P^{(n)} \rightarrow H \underline{\mathbb{Z}}$ (which is the unit map in the category of $B P^{(n)}$-algebras). A simple check on geometric fixed points shows that this map is not null. However, when we compose with the map to $B P^{(n)} \wedge_{B P^{(n)}}$ $H \underline{\mathbb{Z}}$, then this map becomes null.

Definition 7.8. Let $\tau_{i}: S^{\left(2^{i}-1\right) \rho_{G}+1} \rightarrow B P^{(n)} /\left(G \cdot \bar{v}_{i}\right) \wedge_{B P^{(n)}} H \underline{Z}$ denote a preimage of $N\left(\bar{v}_{i}\right)$ in the long exact sequence in homotopy induced by the cofiber sequence.

Let $\tau_{i}: S^{\left(2^{i}-1\right) \rho_{G}+1} \rightarrow H \underline{\mathbb{Z}} \wedge_{B P^{(n)}} H \underline{\mathbb{Z}}$ also denote the image of $\tau_{i}$ into the colimit.

Obviously there is substantial ambiguity in this definition. However, for our purposes we need most understand what happens modulo the elements annihilated by $a_{\sigma}$. For this, the following proposition shows there is not only is there no ambiguity but also the classes are non-zero. is $b^{\prime 2^{i}}$.

Proposition 7.9. The image of $a_{\left(2^{i}-1\right) \bar{\rho}_{G}} \tau_{i}$ in $\pi_{*} \Phi^{G}\left(H \underline{\mathbb{Z}} \wedge_{B P^{(n)}} H \underline{\mathbb{Z}}\right)$ 
Proof. This is immediate from applying geometric fixed points to the defining cofiber sequences.

In fact, this proposition is essentially equivalent to the Reduction theorem. The key step is that we can get $H \underline{\mathbb{Z}}$ from $B P^{(n)}$ by killing classes, and the argument relies on a computation. This computation is at its heart equivalent to knowing that the classes $\tau_{i}$ map to non-trivial classes in $H \underline{\mathbb{Z}} \wedge_{B P^{(n)}} H \underline{\mathbb{Z}}$.

Since this is a map of rings, we note that there are non-trivial multiplicative extensions among the classes $\tau_{i}$.

Corollary 7.10. Modulo elements killed by inverting $a_{\sigma}$, we have a relation

$$
\tau_{i}^{2}=a_{\bar{\rho}_{G}} \tau_{i+1}
$$

For computational reasons, it is convenient to extend this result very slightly and kill 2 in the homotopy. Let $\tau_{0}: S^{1} \rightarrow H \underline{\mathbb{Z}} / 2 \wedge_{B P^{(n)}} H \underline{\mathbb{Z}} / 2$ denote the mod 2 Bockstein.

Proposition 7.11. The image of $\tau_{0}$ in $\pi_{*} \Phi^{G}\left(H \underline{\mathbb{Z}} / 2 \wedge_{B P^{(n)}} H \underline{\mathbb{Z}} / 2\right)=$ $\mathbb{F}_{2}\left[\sqrt{b}, \sqrt{b^{\prime}}\right]$ is $\sqrt{b^{\prime}}$.

Corollary 7.12. for all $i \geq 0$, we have

$$
\tau_{i}^{2}=a_{\bar{\rho}_{G}} \tau_{i+1} .
$$

REMARK 7.13. This result has a number of antecedents. In motivic homotopy theory, we can also form $H \mathbb{F}_{2} \wedge_{B P} H \mathbb{F}_{2}$. Work of Voevodsky shows that the homotopy groups of this are $\pi_{\star} H \mathbb{F}_{2}\left[\tau_{0}, \ldots\right] / \tau_{i}^{2}=\rho \tau_{i+1}$, where $\rho$ is a distinguished class in the motivic homotopy of a point.

The computations in this section have been in homology. We turn now to cohomology to produce our desired differentials. Let $Q_{i}$ denote the class in $\left(B P^{(n)}\right.$-relative) cohomology dual to $\tau_{i}$. This is an element in $\pi_{\star} F_{B P^{(n)}}(H \underline{\mathbb{Z}} / 2, H \underline{\mathbb{Z}} / 2)$, the ring of operations on mod 2-cohomology in the category of $B P^{(n)}$-modules. Since the dual has a product, this has a coproduct, and the multiplicative relations on the classes $\tau_{i}$ gives us a coproduct relations on the classes $Q_{i}$.

Proposition 7.14. The class $a_{\bar{\rho}_{G}} Q_{i-1} \otimes Q_{i-1}$ occurs in the coproduct of $Q_{i}$ with non-zero coefficient.

These classes are not a priori in the equivariant Steenrod algebra. These are classes in the homotopy of the spectrum of $B P^{(n)}$-module endomorphisms of $H \underline{\mathbb{Z}} / 2$. However, since the slice filtration is a filtration of $B P^{(n)}$ modules, this is sufficient for our purposes. In fact, though not needed, it is not difficult to show for naturality reasons that these map to non-trivial elements in the equivariant Steenrod algebra. 
THEOREM 7.15. Modulo classes annihilated by inverting $a_{\sigma}$,

$$
Q_{i}\left(u_{\sigma}^{2^{i}}\right)=a_{\bar{\rho}_{G}}^{2^{i}-1} a_{\sigma}^{2^{i}} .
$$

Proof. The equivariant chain complexes show that $Q_{0}\left(u_{\sigma}\right)=a_{\sigma}$. The result now follows by induction.

THEOREM 7.16. In the slice spectral sequence for $B P^{(n)}$, we have differentials

$$
d\left(u_{2 \sigma}^{2^{i}}\right)=a_{\bar{\rho}_{G}}^{2^{i+1}-1} a_{\sigma}^{2^{i+1}} N\left(\bar{v}_{i}\right) .
$$

Proof. This is immediate from the definition of the classes $\tau_{i}: \tau_{i}$ detects multiplication by $N\left(\bar{v}_{i}\right)$. The previous theorem them gives this result modulo classes annihilated by $a_{\sigma}$. A simple computation shows that in this range of possible targets, multiplication by $a_{\sigma}$ is faithful.

As we shall see, this theorem is the essential result needed for the periodicity and homotopy fixed points theorems.

7.3. Interlude $K_{\mathbb{R}}$ and $K O$. To ground the previous differentials, we present the example of the $C_{2}$-fixed points of $K_{\mathbb{R}}$. This computation was originally done by Dugger with his $C_{2}$-slice spectral sequence $[\mathbf{1 0}]$. We reproduce it with our language and machinery. The $E_{2}$-page of the spectral sequence is shown in Figure 5. We have included the names of several classes to help the reader. In particular, classes that occur on the zero line are powers of $\bar{v}_{1}$ times complementary powers of $u_{2 \sigma}$. As always, boxes are copies of $\mathbb{Z}$ while black dots are copies of $\mathbb{Z} / 2$.

The class $\bar{v}_{1}$ is an actual equivariant homotopy class, so we know it is a permanent cycle in the slice spectral sequence. Similarly, $a_{\sigma}$ is in the Hurewicz image. The only class unaccounted for is $u_{2 \sigma}$, and Theorem 7.16

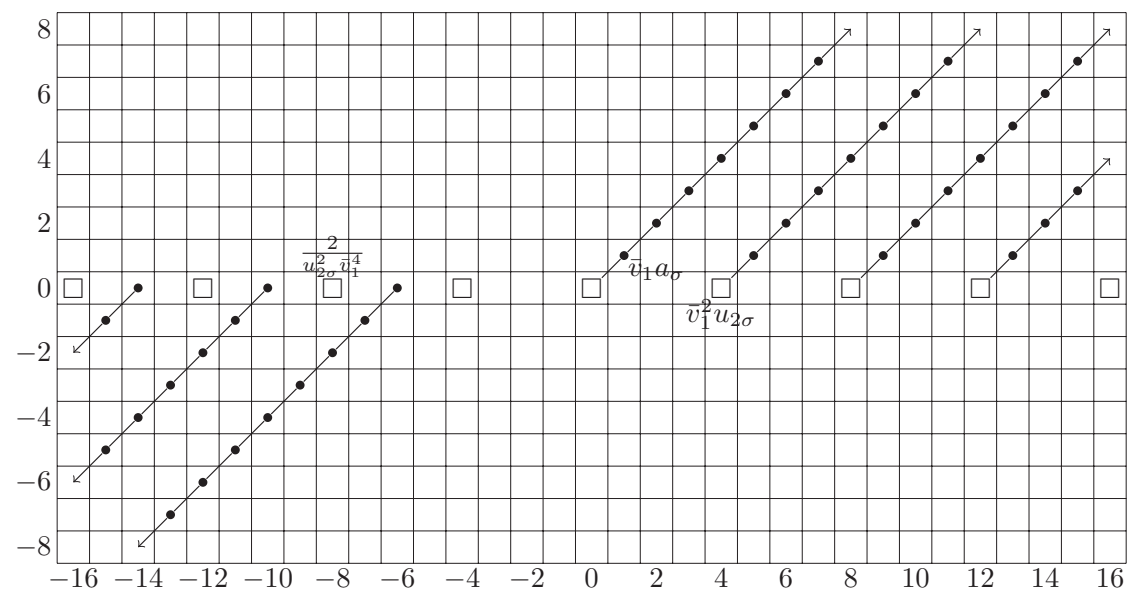

Figure 5. The Slice $E_{2}$-term for $K_{\mathbb{R}}$ 


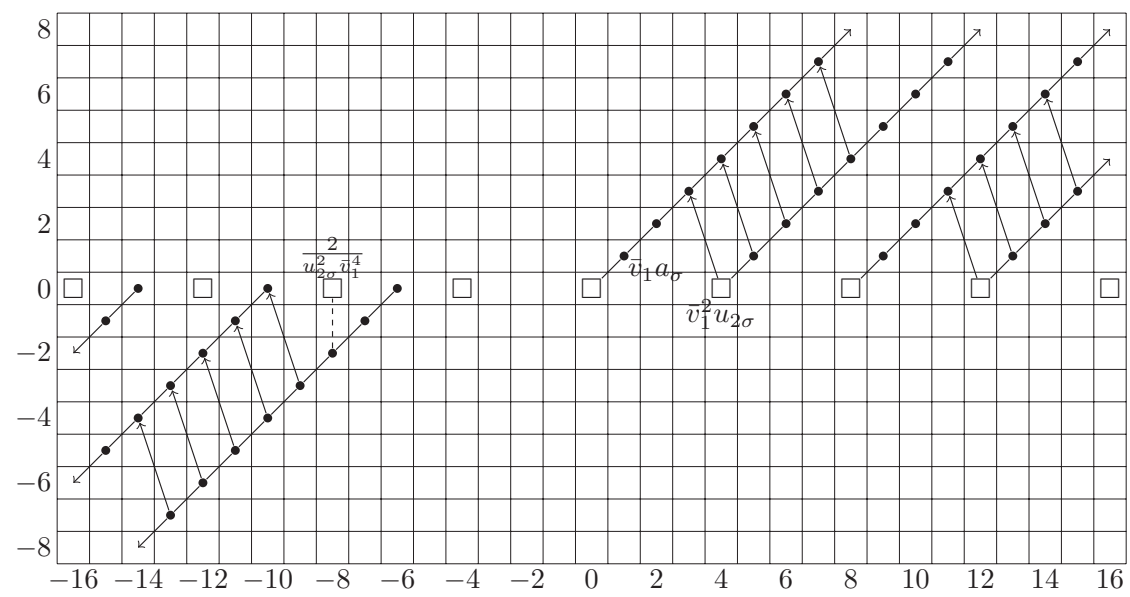

Figure 6 . The Slice $E_{3}$-term for $K_{\mathbb{R}}$

shows that it supports a slice $d_{3}$ :

$$
d_{3}\left(u_{2 \sigma}\right)=a_{\sigma}^{3} \bar{v}_{1} .
$$

This differential takes place in an equivariant stem (connecting the $2-2 \sigma$ and the $1-2 \sigma$ stems). However, since this is a spectral sequence of algebras, we see $\mathbb{Z}$-graded versions of this:

$$
d_{3}\left(\bar{v}_{1}^{2} u_{2 \sigma}\right)=\bar{v}_{1}^{2} d_{3}\left(u_{2 \sigma}\right)=a_{\sigma}^{3} \bar{v}_{1}^{3} .
$$

We present the $E_{3}$-page together with this differential in Figure 6 . The reader is directed to the positive parts of the homotopy, where the slice spectral sequence looks very much like the homotopy fixed points spectral sequence under the line of slope 1 through the origin. In negative degrees, we see essentially the dual pattern, and we will not dwell much on those stems.

For $K_{\mathbb{R}}$, the class $\bar{v}_{1}$ is a unit. This means that we also have a differential

$$
d_{3}\left(\bar{v}_{1}^{-1} u_{2 \sigma}\right)=a_{\sigma}^{3},
$$

and so $a_{\sigma}^{3}=0$ from $E_{4}$ on. This produces a horizontal vanishing line in the spectral sequence, and $E_{4}=E_{\infty}$. In particular (even if $E_{4}$ were not $E_{\infty}$ ), we see that $u_{2 \sigma}^{2}$ is a permanent cycle. We also see that since $a_{\sigma}$ is nilpotent, the geometric fixed points of $K_{\mathbb{R}}$ is contractible. All of this followed simply from the invertibility of $\bar{v}_{1}$, and these types of properties will be key in our identification of the periodicity.

\section{Homotopy fixed points, periodicity, and the spectrum $\Omega$}

In this section, we will complete our sketch, tying our Gap Theorem with the differentials we discovered. In all cases, the classes $N_{2^{k}}^{2^{n}}\left(\bar{r}_{i, k}\right)$ play a special role: they are carried by regular representation spheres and are seen 
by the differentials. Thus upon inverting families of these classes, we get spectra with a gap and for which the slice spectral sequence is simplified. Since these norms will occur quite frequently in all that follows, let

$$
N_{k}^{n}(x)=N_{C_{2^{k}}}^{C_{2^{n}}}(x) \text {. }
$$

8.1. The homotopy fixed point and periodicity theorems. The last results needed for various localizations of $M U_{\left(C_{2^{n}}\right)}$ rely on an interplay between things we can compute for the homotopy fixed points and things we can compute for honest fixed points. We begin with a slightly general theorem that will greatly help us. Let $E G$ denote the free contractible $G$-space. Recall also that we defined spaces $E \mathcal{F}_{m}$ to be the universal space for the family of proper subgroups of $C_{p^{m}}$.

THEOREM 8.1. Let $R$ be a $G$ ring spectrum. If for all $m>0\left(\tilde{E} \mathcal{F}_{m} \wedge\right.$ $R)^{C_{p^{m}}}$ is contractible, then the natural map

$$
R \rightarrow F\left(E G_{+}, R\right)
$$

is a $G$-weak equivalence.

Proof. The proof is by induction on the order of the group. If the the group is trivial, then the result is obviously true. Assume now that for all subgroups of $C_{p^{n}}$ the result is true and that the geometric fixed point object is contractible. If we smash the map $\iota: R \rightarrow F\left(E G_{+}, R\right)$ with the isotropy separation sequence, then we have a diagram

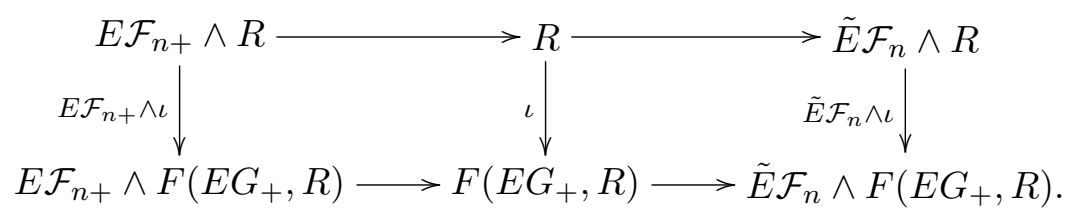

By induction, the map labeled $E \mathcal{F}_{n+} \wedge \iota$ is a $G$-weak equivalence. By the assumption on $R$, the upper right corner is equivariantly contractible. The map labeled $\tilde{E} \mathcal{F}_{n} \wedge \iota$ is a ring map, so we learn that it must also be an equivalence. Therefore the map labeled $\iota$ is an equivalence.

The results of the previous section show us exactly how to ensure that these geometric fixed points sets are contractible for various $M U_{\left(C_{2^{n}}\right)^{-}}$ algebras.

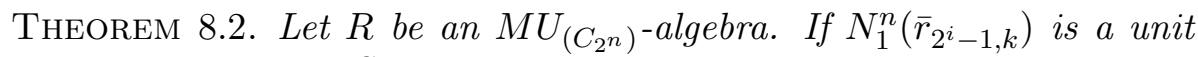
in $\pi_{\star} R$, then $\left(\tilde{E} \mathcal{F}_{k} \wedge R\right)^{C_{2^{k}}}$ is contractible.

Proof. Since we are computing the homotopy groups of the $C_{2^{k}}$-fixed points, by naturality of the slice spectral sequence we may assume that $k=n$ and show the result there.

Theorem 7.16 show that we have a slice differential

$$
u_{2 \sigma_{n}}^{2^{i-1}} \mapsto a_{\bar{\rho}}^{2^{i}-1} a_{\sigma}^{2^{i}} N_{2}^{n}\left(\bar{r}_{2^{i}-1, n}\right) .
$$


Since $N_{2}^{n}\left(\bar{r}_{2^{i}-1, n}\right)$ is a unit, this allows us to conclude that starting on the next page of the spectral sequence $a_{\sigma}^{2^{i}+1} a_{\bar{\rho}}^{2^{i}-1}=0$. Simple computations with the complexes occurring in the slice tower show that there are not possible targets for a hidden $a_{\sigma}$-multiplication (as this class is always at the farthest edge of the vanishing region), so we conclude that after inverting $a_{\sigma}$, we get the zero ring. This gives the result.

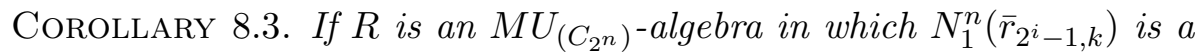
unit, then $u_{2 \sigma_{k}}^{2^{i}}$ is a permanent cycle.

Proof. Again, the statement is one taking place for $C_{2^{k}}$ fixed points, so we may assume $k=n$. If we smash the slice tower of $M U_{\left(C_{2^{n}}\right)}$ with $S^{2^{j} \sigma}$ and take homotopy, then we get a spectral sequence computing $\pi_{*}$ $\left(\Sigma^{2^{j} \sigma} M U_{\left(C_{2}\right)}\right)$. Using our complexes, it is easy to see that the last possible differential on $u_{2 \sigma}^{2^{j-1}}$ (which indeed is the only possible differential) is the one we found using power operations.

Since under the hypothesis the target of the differential on $u_{2 \sigma}^{2^{i}}$ is obviously already killed, we conclude that $u_{2 \sigma}^{2^{i}}$ is a permanent cycle.

These two results are the heart of the Homotopy Fixed Point and Periodicity theorem.

Theorem 8.4 (Homotopy Fixed Point and Periodicity Theorems). Assume given a sequence of non-negative numbers $i_{1}$ through $i_{n}$, let $i$ be the maximum of the $i_{j}$, let $i^{\prime}$ denote the minimum of the $i_{j}$, and assume $\bar{\Delta}: S^{m \rho} \rightarrow M U_{\left(C_{2^{n}}\right)}$ is divisible by $N_{1}^{n}\left(\bar{r}_{2^{i} k-1, k}\right)$ for all $k$. Then

(1) We have a weak equivalence

$$
\left(\bar{\Delta}^{-1} M U_{\left(C_{2^{n}}\right)}\right)^{C_{2^{n}}} \simeq\left(\bar{\Delta}^{-1} M U_{\left(C_{2^{n}}\right)}\right)^{h C_{2^{n}}} .
$$

(2) The class $u_{2 \rho}^{2^{i}}$ lifts to a non-zero homotopy class in $\bar{\Delta}^{-1} M U_{\left(C_{2^{n}}\right)}$.

(3) $\pi_{*} \bar{\Delta}^{-1} M U_{\left(C_{2}\right)}$ is $2^{n} \cdot \operatorname{lcm}\left(2^{i+1},\left(2^{i^{\prime}}-1\right)\right)$-periodic.

While not immediately obviously so, all three parts of this result are intimately related and are simple consequences of our earlier theorems. For this reason, we link them into a single result

Proof. The proof of the first part is immediate from Theorems 8.1 and 8.2. For the second part, we know from Corollary 8.3 that for all $k, u_{2 \sigma_{k}}^{2^{i}}$ is a permanent cycle and therefore a homotopy class. This means that for all $k N_{k}^{n}\left(u_{2 \sigma_{k}}^{2^{i}}\right)$ is a permanent cycle and therefore a homotopy class, and so multiplying these classes gives

$$
u_{2 \rho}^{2^{i}}=\prod_{k=1}^{n} N_{k}^{n}\left(u_{2 \sigma_{k}}^{2^{i}}\right)
$$

is a permanent cycle and a homotopy class. 
The third result combines the first two. The class $u_{2 \rho}^{2^{i}}$ gives an equivariant map

$$
\Sigma^{2^{i+n+1}} \bar{\Delta}^{-1} M U_{\left(C_{2^{n}}\right)} \rightarrow \Sigma^{2^{i+1} \rho} \bar{\Delta}^{-1} M U_{\left(C_{2^{n}}\right)},
$$

and when we restrict to the trivial group, this map is the identity. Thus we have an equivariant map that is an underlying equivalence, and that gives us an equivalence on homotopy fixed points. Since these are the same as honest fixed points, we conclude that the class $u_{2 \rho}^{2^{i}}$ is invertible in $\pi_{\star} \bar{\Delta}^{-1} M U_{\left(C_{2^{n}}\right)}$. The desired periodicity arises by multiplying $u_{2 \rho}^{2^{i}}$ by the appropriate powers of $N_{k}^{n}\left(\bar{r}_{2^{i} k-1, k}\right)$ as $k$-varies (all of which will be invertible classes in trivial stems). If we chose $i_{k}$ to be minimal, then we get the smallest predictable periodicity.

Since $\bar{\Delta}$ in the previous theorem is carried by a regular representation sphere, we also conclude that $\pi_{-2} \bar{\Delta}^{-1} M U_{\left(C_{2} n\right)}=0$. Since the homotopy groups are periodic, this shows that a large number of groups are actually zero. We should stress here the importance of the Homotopy Fixed Point theorem. The Detection ad Periodicity Theorems are proved for the homotopy fixed points of $\tilde{\Omega}$, not the honest fixed points. On the other hand, the Gap Theorem is proved for honest fixed points which a priori only map to the homotopy fixed points. The Homotopy Fixed Point Theorem provides the bridge between the two kinds of results.

8.2. The spectrum $\Omega$ and the detection theorem. As was described early on, our spectrum $\tilde{\Omega}$ is a stand-in for $E_{4}$ with the $C_{8}$-action. We will build $\tilde{\Omega}$ by inverting equivariant classes for each of the subgroups of $C_{8}$, just as in the Homotopy Fixed Points Theorem, while ensuring that we have good equivariant maps from these localizations to $E_{4}$.

We first look at the underlying spectrum for $M U_{C_{8}}$. This is $M U \wedge M U \wedge$ $M U \wedge M U$ with the permutation action of $C_{8}$, and the underlying homotopy groups represent the data of having 4 formal group laws $F_{1}, \ldots, F_{4}$ together with a chain of isomorphisms

$$
F_{1} \stackrel{\gamma}{\rightarrow} F_{2} \stackrel{\gamma}{\rightarrow} F_{3} \stackrel{\gamma}{\rightarrow} F_{4}
$$

such that $\gamma^{4}=[-1](x)$. In particular, we have a canonical map

$$
\pi_{*}(M U \wedge M U \wedge M U \wedge M U) \rightarrow \pi_{*} E_{4}
$$

that classifies the universal deformation formal group law carried by $E_{4}$, together with its translates under the group $C_{8}$. This then gives us by construction a $C_{8}$-equivariant map

$$
M U \wedge M U \wedge M U \wedge M U \rightarrow E_{4} .
$$

Through this map, we will isolate equivariant elements which can be safely inverted without destroying the detection of the Kervaire classes.

In particular, we have the following result, since $E_{4}$ is $v_{4}$-periodic and since $r_{15,1} \equiv v_{4}$ modulo the previous classes. 
Proposition 8.5. The map

$$
M U \wedge M U \wedge M U \wedge M U \rightarrow E_{4}
$$

factors through $\left(N_{1}^{3} \bar{v}_{4}\right)^{-1} M U \wedge M U \wedge M U \wedge M U$.

To fully spell out the reasoning for inverting the classes we do, we must have a brief digression into the Morava stabilizer group $\mathbb{S}_{4}[\mathbf{2 5}]$. By definition, this is the automorphism group of the Honda formal group law, and it can be realized as the group of units of the maximal order of the division ring over $\mathbb{Q}_{2}$ of Hasse invariant $1 / 4$. In particular, using a presentation of this division ring, we can write any element $g \in \mathbb{S}_{4}$ as

$$
g=\sum_{j=0}^{\infty} t_{j}\left(g^{-1}\right) T^{j},
$$

where $t_{j}\left(g^{-1}\right)$ is an element of $\mathbb{F}_{15}$. As is suggested by the notation, the symbols $t_{j}$ are functions on the Morava stabilizer group, and they are also the images of the elements by the same name in $B P_{*} B P$. Thus this formula reflects the interplay between the $B P_{*} B P$-coaction and the group action. For us, the most important part is that we can check if the value of $t_{i}$ on a group element is a non-zero element of $\pi_{*} E_{4}$ modulo the maximal ideal. Since $\pi_{*} E_{4}$ is a complete local ring, any element in $\pi_{*} E_{4}$ that has non-zero reduction modulo the maximal ideal is a unit. Restricting attention to $C_{4}$, this gives us another class we can invert, since an elementary argument involving valuations shows that $t_{2}\left(\gamma^{2}\right) \neq 0$. Modulo lower classes, $t_{2} \equiv r_{3,2}$.

Proposition 8.6. The map

$$
M U \wedge M U \wedge M U \wedge M U \rightarrow E_{4}
$$

factors through $\left(N_{1}^{3} \bar{r}_{3,2}\right)^{-1} M U \wedge M U \wedge M U \wedge M U$.

As stated, the result about the interplay between the elements $t_{i}$ and the group action is for $M U \wedge M U$ (really $B P \wedge B P$ ). We are working with $M U \wedge M U \wedge M U \wedge M U$. However, a similar analysis shows that we have the same relationship between the classes $r_{2^{i}-1,3}$ and the generator $\gamma$ (in fact, this is essentially a way we could define $\left.r_{1,3}\right)$. The same valuation argument use for $C_{4}$ shows that $r_{1,3}(\gamma) \neq 0$.

Proposition 8.7. The map

$$
M U \wedge M U \wedge M U \wedge M U \rightarrow E_{4}
$$

factors through $\left(N_{1}^{3} \bar{r}_{1,3}\right)^{-1} M U \wedge M U \wedge M U \wedge M U$.

At this point, we can no longer say if there are any elements we can obviously invert. We have inverted appropriate norm classes for each subgroup, and we know that the resulting map has an equivariant map from the underlying spectrum to $E_{4}$. We can therefore define $\tilde{\Omega}$. 
DEFINITION 8.8. Let $\tilde{\Omega}$ be

$$
\left(N_{1}^{3} \bar{r}_{1,3}\right)^{-1}\left(N_{1}^{3} \bar{r}_{3,2}\right)^{-1}\left(N_{1}^{3} \bar{r}_{15,1}\right)^{-1} M U_{C_{8}} .
$$

Recall that $\Omega$ was defined to be the fixed points of $\tilde{\Omega}$. An immediate application of our big theorems shows the following.

\section{COROLlary 8.9 .}

(1) The homotopy groups of $\Omega$ are 256-periodic.

(2) We have $\pi_{-2} \Omega=0$.

(3) We have an equivalence $\Omega \simeq \tilde{\Omega}^{h C_{8}}$.

These are exactly the properties needed to prove the Kervaire theorem. We state without proof the final piece. The argument for this is classical: we show using the homotopy fixed point spectral sequence and the comparison with the Adams-Novikov spectral sequence that any element representing the Kervaire classes maps to a non-zero element. Since the argument is of a decidedly different flavor, we refer the reader to the main paper.

TheOREM 8.10 (Detection Theorem). If $\theta_{j} \in \pi_{2^{j+1}-2} S^{0}$ is non-zero, then it has non-zero Hurewicz image in $\pi_{*} \Omega$.

Since these elements are then detected in the zero group for $j \geq 7$, we conclude that these elements do not survive.

\section{References}

[1] Shôrô Araki. Coefficients of $M R$-theory. Available online at www.math.rochester. edu/u/faculty/doug/.

[2] Shôrô Araki. Orientations in $\tau$-cohomology theories. Japan. J. Math. (N.S.), 5(2): 403-430, 1979.

[3] M. F. Atiyah. K-theory and reality. Quart. J. Math. Oxford Ser. (2), 17:367-386, 1966.

[4] Maia Averett. Completion of Real Johnson-Wilson theory $E \mathbb{R}(n)$ gives fixed points of Morava E-theory. PhD thesis, UCSD.

[5] G. Carlsson. A survey of equivariant stable homotpy theory. Topology, 31:1-28, 1992.

[6] Ethan S. Devinatz, Michael J. Hopkins, and Jeffrey H. Smith. Nilpotence and stable homotopy theory. I. Ann. of Math. (2), 128(2):207-241, 1988.

[7] T. tom Dieck. Kobordismtheorie klassifiziender Räume und Transformationsgrupen. Mathematische Zeitschrift, 126:31-39, 1972.

[8] T. tom Dieck. Transformation Groups and Representation Theory, volume 766 of Lecture Notes in Mathematics. Springer-Verlag, New York, 1979.

[9] Andreas W. M. Dress. Contributions to the theory of induced representations. In $\mathrm{Al}$ gebraic K-theory, II: "Classical" algebraic K-theory and connections with arithmetic (Proc. Conf., Battelle Memorial Inst., Seattle, Wash., 1972), pages 183-240. Lecture Notes in Math., Vol. 342. Springer, Berlin, 1973.

[10] Daniel Dugger. An Atiyah-Hirzebruch spectral sequence for $K R$-theory. $K$-Theory, 35(3-4):213-256 (2006), 2005.

[11] Leonard Evens. A generalization of the transfer map in the cohomology of groups. Trans. Amer. Math. Soc., 108:54-65, 1963.

[12] E. Dror Farjoun. Cellular spaces, null spaces and homotopy localization, volume 1622 of Lecture Notes in Mathematics. Springer-Verlag, New York, 1996. 
[13] J. P. C. Greenlees and J. P. May. Equivariant stable homotopy theory. In Handbook of algebraic topology, pages 277-323. North-Holland, Amsterdam, 1995.

[14] J. P. C. Greenlees and J. P. May. Localization and completion theorems for $M U-$ module spectra. Ann. of Math. (2), 146(3):509-544, 1997.

[15] Michael A. Hill, Michael J. Hopkins, and Douglas C. Ravenel. On the non-existence of elements of Kervaire invariant one. Available on the arxiv, 2009.

[16] Michael J. Hopkins and Fabien Morel. On the zero slice of $M G L$ and $S^{0}$. In preparation.

[17] Po Hu and Igor Kriz. Real-oriented homotopy theory and an analogue of the AdamsNovikov spectral sequence. Topology, 40(2):317-399, 2001.

[18] Nitu Kitchloo and W. Stephen Wilson. On the Hopf ring for ER(n). Topology Appl., 154(8):1608-1640, 2007.

[19] Peter S. Landweber. Conjugations on complex manifolds and equivariant homotopy of MU. Bull. Amer. Math. Soc., 74:271-274, 1968.

[20] L. G. Lewis, J. P. May, and M. Steinberger. Equivariant Stable Homotopy Theory, volume 1213 of Lecture Notes in Mathematics. Springer-Verlag, New York, 1986.

[21] L. Gaunce Lewis, Jr. The $R \mathrm{O}(G)$-graded equivariant ordinary cohomology of complex projective spaces with linear $\mathbf{Z} / p$ actions. In Algebraic topology and transformation groups (Göttingen, 1987), volume 1361 of Lecture Notes in Math., pages 53-122. Springer, Berlin, 1988.

[22] M. A. Mandell and J. P. May. Equivariant orthogonal spectra and $S$-modules. Mem. Amer. Math. Soc., 159(755):x+108, 2002.

[23] M. A. Mandell, J. P. May, S. Schwede, and B. Shipley. Model categories of diagram spectra. Proc. London Math. Soc. (3), 82(2):441-512, 2001.

[24] J. P. May. Equivariant homotopy and cohomology theory, volume 91 of CBMS Regional Conference Series in Mathematics. Published for the Conference Board of the Mathematical Sciences, Washington, DC, 1996. With contributions by M. Cole, G. Comezaña, S. Costenoble, A. D. Elmendorf, J. P. C. Greenlees, L. G. Lewis, Jr., R. J. Piacenza, G. Triantafillou, and S. Waner.

[25] Douglas C. Ravenel. Complex cobordism and stable homotopy groups of spheres, volume 121 of Pure and Applied Mathematics. Academic Press Inc., Orlando, FL, 1986. Errata and second edition available online at author's home page.

[26] Charles Rezk. Notes on the Hopkins-Miller theorem. In Homotopy theory via algebraic geometry and group representations (Evanston, IL, 1997), volume 220 of Contemp. Math., pages 313-366. Amer. Math. Soc., Providence, RI, 1998.

[27] V. Voevodsky. On the zero slice of the sphere spectrum. Tr. Mat. Inst. Steklova, 246(Algebr. Geom. Metody, Svyazi i Prilozh.):106-115, 2004.

[28] Vladimir Voevodsky. Open problems in the motivic stable homotopy theory. I. In Motives, polylogarithms and Hodge theory, Part I (Irvine, CA, 1998), volume 3 of Int. Press Lect. Ser., pages 3-34. Int. Press, Somerville, MA, 2002.

[29] Vladimir Voevodsky. A possible new approach to the motivic spectral sequence for algebraic K-theory. In Recent progress in homotopy theory (Baltimore, MD, 2000), volume 293 of Contemp. Math., pages 371-379. Amer. Math. Soc., Providence, RI, 2002 .

[30] Klaus Wirthmüller. Equivariant homology and duality. Manuscripta Math., 11: 373-390, 1974.

[31] Michael A. Hill, Michael J. Hopkins, and Douglas C. Ravenel. The Arf-Kervaire invariant problem in algebraic topology: introduction. In Current developments in mathematics, 2009, pages 23-57, Int. Press, Somerville, MA, 2010. 
